\title{
Dynamics of Cross Flow Heat Exchanger Tubes with Multiple Loose Supports
}

\author{
K.T. Anwar Sadath
}

\author{
A Thesis Submitted to \\ Indian Institute of Technology Hyderabad \\ In Partial Fulfillment of the Requirements for \\ The Degree of Master of Technology
}

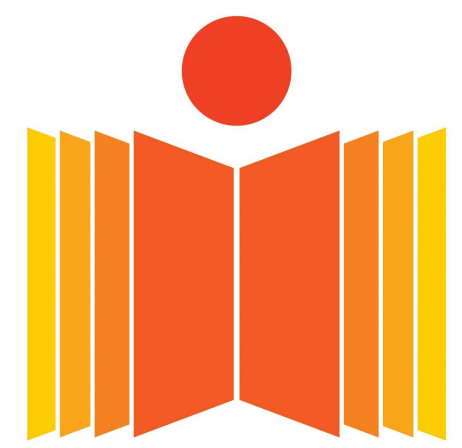

भारतीय प्रौद्योगिकी संस्थान हैदराबाद

Indian Institute of Technology Hyderabad

Department of Mechanical and Aerospace Engineering 


\section{Declaration}

I declare that this written submission represents my ideas in my own words, and where ideas or words of others have been included, I have adequately cited and referenced the original sources. I also declare that I have adhered to all principles of academic honesty and integrity and have not misrepresented or fabricated or falsified any idea/data/fact/source in my submission. I understand that any violation of the above will be a cause for disciplinary action by the Institute and can also evoke penal action from the sources that have thus not been properly cited, or from whom proper permission has not been taken when needed.

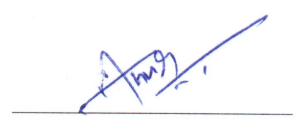

(Signature)

(K.T. Anwar Sadath)

(Roll No. ME12M14P000001) 


\section{Approval Sheet}

This Thesis entitled Dynamics of Cross Flow Heat Exchanger Tubes with Multiple Loose Supports by K.T. Anwar Sadath is approved for the degree of Master of Technology from IIT Hyderabad

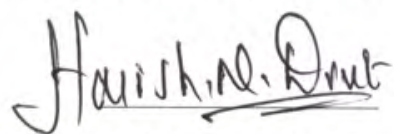

(Dr. Harish N Dixit) Examiner

Dept. of Mechanical and Aerospace Engineering, IITH

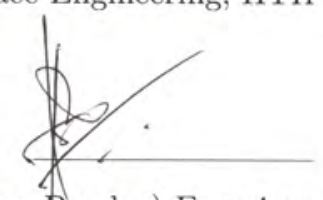

(Dr. Ashok Kumar Pandey) Examiner

Dept. of Mechanical and Aerospace Engineering, IITH

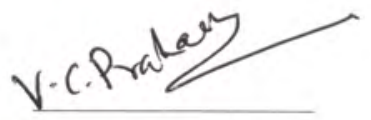

(Dr. Chandrika Prakash Vyasarayani) Adviser

Dept. of Mechanical and Aerospace Engineering, IITH

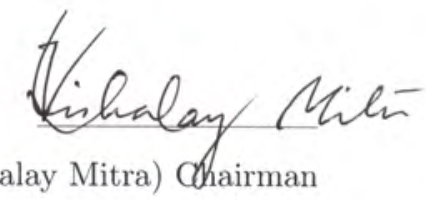

(Dr. Kishalay Mitra) Chairman

Dept. of Chemical Engineering, IITH 


\section{Acknowledgements}

I would like to express my gratitude to all those who helped me to finish my thesis. First and foremost I would like to thank God, the almighty, for giving me strength and courage to do research.

I would like to thank my parents and siblings for their love, endless support and encouragement.

I express my sincere gratitude to my supervisor Dr. V. C. Prakash for his inspiration, valuable guidance, timely suggestions and constant encouragement during each and every phase of this work.

Also I would like to extend my gratitude to Dr. R. Viswanath for assisting me in the the selection of topic and for valuable guidance.

Finally, I would like to thank all my friends Vinu, Zaid, Quadir, Harilal, Ali, and Nikhil for supporting me to finish my work. 


\section{Dedication}

This thesis is dedicated to my parents \& siblings for their love, endless support and encouragement 


\begin{abstract}
Dynamics of cross flow heat exchanger tubes with two loose supports has been studied. The analytical model of a cantilever beam that include the time delayed displacement term along with two restrained spring forces has been used to model the flexible tube. The model consists of one loose support placed at the free end of the beam and the other at the mid-span of the beam. The critical flow has been obtained after solving a free vibration model of the system. The beam equation is discretized to five second order delay differential equations using Galerkin approximation, and solved numerically. It has been found that, for flow velocity less than the critical flow velocity the system shows a positive damping leading to a stable response. Beyond the critical velocity, the system becomes unstable, but a further increase in the velocity leads to the formation a negative damping which stabilize the system at an amplified oscillatory state. For a sufficiently high flow velocity the tube impacts on the loose supports and generates more complex and chaotic vibrations. The loose supports have been modelled as a cubic spring and a tri-linear spring. The effect of spring constants and free-gap of the loose support on the dynamics of the tube has been studied.
\end{abstract}




\section{Contents}

Declaration ...................................... ii

Approval Sheet . . . . . . . . . . . . . . . . . . iii

Acknowledgements . . . . . . . . . . . . . . . . . . . iv iv

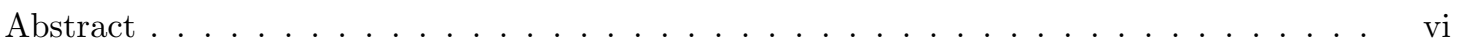

$\begin{array}{lc}\text { Nomenclature } & \text { viii }\end{array}$

1 Introduction $\quad 1$

1.1 Literature review . . . . . . . . . . . . . . . . . . . . . . . . 3

1.2 Problem definition . . . . . . . . . . . . . . . . . . . . . 5

1.3 Thesis structure . . . . . . . . . . . . . . . . . . 5

2 The flow-induced forces on a flexible tube 6

2.1 Flow retardation . . . . . . . . . . . . . . . . . . . 7

2.2 Drag and lift force on cylinder due to fluid flow . . . . . . . . . . . . . . . . 7

2.3 Fluid forces induced by impulsive body motion . . . . . . . . . . . . . . . 8

2.4 Resultant fluid forces acting on a tube in a tube array along the lift direction . . . . 9

3 Modelling of a flexible tube subjected to cross flow 10

3.1 The equation of motion of the beam . . . . . . . . . . . . . . . . . . 10

3.2 Non-dimensionalisation . . . . . . . . . . . . . . . . . . . . . 11

3.3 Discretization using Galerkin approximation . . . . . . . . . . . . . . . . . . 13

3.4 Generalized equation of motion in first-order form . . . . . . . . . . . . . . . . 13

3.5 Numerical solution . . . . . . . . . . . . . . . . . . . . . . . . 14

4 Fixed-fixed beam modelling $\quad 15$

4.1 Shape functions . . . . . . . . . . . . . . . . . . . . . . 15

4.2 Hopf bifurcation . . . . . . . . . . . . . . . . . . . . . 15

4.3 Constrained force modelling . . . . . . . . . . . . . . . . . . . . . . . . 17

4.4 Modelling of baffle as a cubic spring . . . . . . . . . . . . . . . . . . . . 17

4.4.1 Bifurcation due to symmetric loading . . . . . . . . . . . . . 18

4.4 .2 Bifurcation due to asymmetric loading . . . . . . . . . . . . . . . 19

4.5 Modelling of baffle as a tri-linear spring . . . . . . . . . . . . . . . . . . . 22

4.5.1 Bifurcation due to symmetric loading . . . . . . . . . . . . . 25

4.5.2 Bifurcation due to asymmetric loading . . . . . . . . . . . . . 25 
5 Fixed-free beam modelling

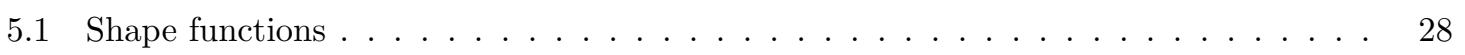

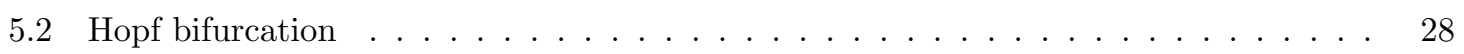

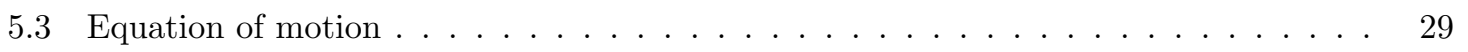

5.4 Loose supports as a cubic spring . . . . . . . . . . . . . . . . . . 30

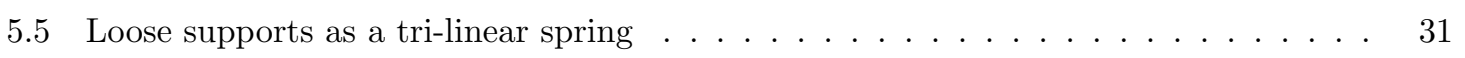

$\begin{array}{lll}6 & \text { Conclusions } & 37\end{array}$

$\begin{array}{ll}\text { References } & 39\end{array}$ 


\section{Chapter 1}

\section{Introduction}

Fluid structure interaction (FSI) is responsible for generating vibrations in elastic structures. FSI can sometime lead to instabilities that will cause large violent oscillations in these structures. This instability is referred as fluid elastic instability (FEI) in the literature dealing with flow induced vibrations of heat exchanger tubes. In aircraft wing stability problems while in flight the instability is referred as flutter. The instability due to FSI in transmission cables is known as galloping.

In this thesis we are interested to study the FEI in heat exchanger tubes. In a typical cross flow heat exchanger (see Fig. (1.1)), the tube bundles carry the primary fluid and the the secondary fluid is circulated across the tubes. For better heat transfer due to convection, the velocity of the secondary fluid should be reasonably high. However, at a critical velocity, due to FSI, the tubes can encounter FEI. In heat exchanger tubes due to FSI, mainly three types of excitations $[1,2,3,4]$ are possible that can trigger vibrations. These include, turbulence, vortex shedding, and FEI [5]. Usually the vibration generated due to turbulence and vortex shedding is well controlled by the inherent damping present in the structure. However, when FEI is encountered the damping in the structure become positive, that means the damping forces will not stabilize the structure, instead they destabilize it. Once this instability is encountered, the structure will start to oscillate with large amplitudes which can cause fatigue. In heat exchanger tubes it is a common practice to provide gaps at the baffle plates and supports to allow for thermal expansion [6]. When the amplitude of tubes increases after FEI, they start to collide with the supports and baffle plates and this leads

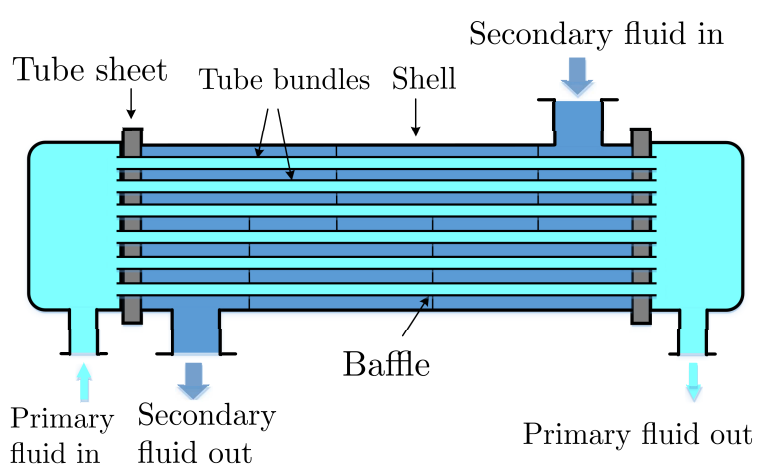

Figure 1.1: Typical shell and tube heat exchanger 


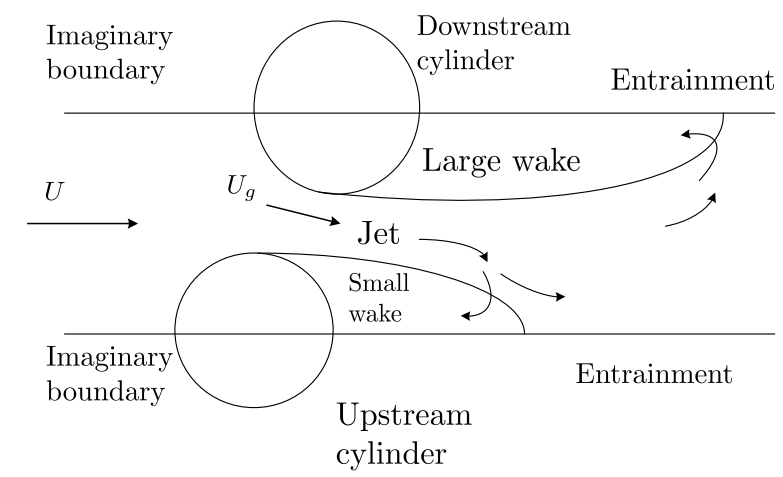

Figure 1.2: Jet-switching model

to fretting failure. In some critical applications like nuclear power plants, such a failure will be disastrous because the radioactive and non-radioactive fluids will get combined and can lead to radiation related medical issues or in worst case can cause explosions. In less critical applications like steam based heat exchangers where the primary fluid and secondary fluids are water, a failure of heat exchanger will cause the power plant to shut down and will cause economical losses to the power plant company.

The post FEI vibratory behavior of these tubes is highly nonlinear because of two reasons, firstly due to large amplitude vibrations, geometrical non linearity of the structure will come into play. Secondly the vibro-impacting motions between the tube and the baffle plates or supports will give rise to impact non-linearity. It is important to understand the nonlinear dynamics of these tubes to obtain the frequency content of the resulting motion. This frequency information can be used for estimating the fatigue life of the tubes after encountering FEI. The FEI is complex phenomena and several researchers developed mathematical models for modelling it. Two popular and simple models are discussed below:

- Jet-switching model: Roberts [7] proposed a semi-analytical jet-Switching model to study FEI in cross flow heat exchanger tubes. Based on some preliminary experiments, it was found that instability is purely in the inflow direction, with adjacent tubes moving out-of-phase with each other. Later he found that the flow downstream of two adjacent tubes can be represented by two wake regions and assumed a jet flow in between a large wake and a small wake as shown in Fig. (1.2). He demonstrated that how a self sustained oscillations can be induced in a tube. When a downstream cylinder moves upstream, the fluid enters into the large wake, thereby the large wake starts to shrink and jet starts to switch directions. When the drag force which helps the downstream motion is greater than that opposing the upstream motion, enough pressure difference is created for jet switching. When the velocity of the secondary fluid is sufficiently high [7] jet-switching will take place.

- Critical velocity model due to Connors and Blevin: Connors $[8,9]$ proposed a model to predict critical velocity of secondary fluid for initiating FEI for tubes in heat exchanger. For a cylinder with mass per unit length $m$ (including the mass of fluid through the tube), diameter $D$, natural frequency $f_{n}$, logarithmic decrement $\delta$, and with $\rho$ being the density of the secondary fluid, Connors proposed a critical velocity $V_{p c}$ at which FEI will initiate. The 
expression for critical velocity $V_{p c}$ is give by:

$$
\frac{V_{p c}}{f_{n} D}=K\left(\frac{m \delta}{\rho D^{2}}\right)^{n}
$$

By assuming that the fluid force acting on the cylinder is due to the relative displacement between cylinder and adjacent cylinder, Blevins [10] improved Conners formula (Eq. (1.1)). Later Blevins modified his own analysis to account for flow-dependent damping. He suggested that for a quasi steady approach, the total damping factor in inflow direction $\left(\zeta_{x}\right)$ and transverse direction $\left(\zeta_{y}\right)$ has to be considered [11]. According to Blevin, the critical velocity is given by:

$$
\frac{V_{p c}}{f_{n} D}=K\left(\frac{m}{\rho D^{2}} 2 \pi \sqrt{\left(\zeta_{x} \zeta_{y}\right)}\right)^{n}
$$

A large number of numerical and experimental studies are reported in the literature dealing with FEI of flexible tubes. The models used for the tubes range from simple single degree of freedom vibration models to more complicated continuous models of flexible beams. Similarly, the fluid motion can be modelled using quasi steady approach or by solving the full Navier-Stokes equation. Ideally the structural motion of the tubes and the Navier-Stokes should be coupled to simulate the full FSI problem of tube and fluid interaction. In the next section we review the literature dealing with FEI in cross flow heat exchanger tubes.

\section{$1.1 \quad$ Literature review}

Ibrahim [6] recently (in the year 2011) wrote an excellent review paper on the topic of FEI in crossflow heat exchanger tubes. An interested reader is referred to his paper for a detailed review of literature dealing with several topics realted to FEI in heat exchanger tubes. In this section, we only review papers that are most relevant to our work. In order to analyse three dimensional flow distribution in heat exchangers, Wambsganss et al [12] developed a three dimensional hydraulic analysis code. For an industrial shell-and-tube exchanger, based on experimental observations, an empirical formula has been developed by Halle et al [13, 14] relating the tube vibration and the velocity of the cross flow. Chen $[15,16]$ experimentally determined the fluid force coefficients for a row of cylinders in square array, and the critical flow velocities were calculated as a function of system parameters. A time-domain forcing function equivalent to Connors [8, 9] equation has been developed by Sauve [17]. Eisinger et al [18] developed a finite element code in ABAQUS to simulate the FEI of non-linear tube bundles. Most of the studies related to heat exchanger tubes are based on axisymmetrically flexible tube bundles, in those cases the instability occurs in transverse direction to the flow. Violette et al [19] conducted a series of tests to study the FEI of cluster tubes. These tubes are more flexible in flow direction than transverse direction. They found that the FEI occurs at higher velocities only when the tubes are flexible in the flow direction.

To incorporate the vibration mechanisms due to vortex shedding, turbulent excitation, and FEI, Yetisir [20] and Weaver [21] developed an unsteady analytical model for fluid forces. The damping decay of disturbance was accounted by introducing a perturbation decay function. Taylor et al [22] conducted a series of tests on multi-span heat exchangers in the air and in water at three different temperatures $\left(25^{\circ} \mathrm{C}, 60^{\circ} \mathrm{C}\right.$, and $\left.90^{\circ} \mathrm{C}\right)$. They found that damping consists of mainly three 
components, viscous damping along the tube, friction damping at the support and squeeze-film damping at the support. They concluded from the experiments that the damping does not change with the temperature and friction damping mainly depends on preload.

Rzentkowski et al [23] studied the effect of turbulence on FEI in tube bundles. The study was for a nonlinear flexible tube surrounded by rigid bundles and constrained to move in the transverse direction of flow. The analysis showed that turbulence of the flow can reduce the FEI limit (in terms of critical velocity) near an unstable bifurcation. However, near a stable bifurcation, the FEI limit was unaffected by turbulence. Weaver [24] found that clipping of the tubes in the heat exchanger may stabilize the system, also longitudinal clipping of three, four, or six tubes together increased the FEI limit.

Vibrations of long flexible tubes can be arrested by using baffles. It is a common practice to include small gaps between the tubes and baffles to allow for thermal expansion of the tubes. These gaps makes the system unstable at low velocities by exciting low frequency modes, this further develops as an instability due to negative damping.

To understand the nonlinear dynamics of tube impacting motions due to loose supports, Jacquart and Gay developed [25] a numerical code named Electricite de France (EDF). They have validated their numerical results with experiments. The nonlinear model of the vibro-impact of heat exchanger tubes was validated by Antunes et al [26] experimentally. Later these experiments were modified by Vento et al [27] to study a two-dimensional motion of tubes by making a circular-support clearance at the mid-span of the tube. They found that the tube vibration depends on the instability level, initial conditions, and tube-support eccentricity. They found that due to friction between the tube and supports, the motion of the tube becomes planar after some impacts.

There are lot of computational methods available to predict the stability of vibro-impact oscillations. A dynamic-finite-element code (VIBIC) has been developed by Rogers et al [28, 29], that can be used to predict the baffle contact forces of a single heat exchanger tube. A nonlinear analytical study was done by Cai et al [30] based on unsteady flow-theory to study chaotic vibration of the tube. Païdoussis et al [31] assumed that among all the tubes only one tube as flexible and studied its nonlinear dynamics. They found that, in a single loose support, the impact was occurring only when the velocity was above the threshold value of FEI. The oscillation becomes highly chaotic for a sufficiently high velocity. Based on the vibrational energy contained in a nuclear steam generator tubes, Fluit and Pettigrew [32] proposed a method to predict the fretting wear damage. This method is also used for analyzing the effect of loose support clearances on the fretting-wear rate.

Using analytical method Païdoussis et al [33] studied the chaotic vibration of heat exchanger tube impacting on loose support. They have included a delayed term to displacement. This entire study was based on a single flexible tube supported as a fixed-fixed beam with a loose support at the centre. Two different types of baffle models were considered, a cubic spring model and a tri-linear spring model. The results shows that the amplitude of oscillation grows after a critical velocity, and the tube starts to impact on the loose support. With further increase in the velocity a more complex motion of the tube is found and for a sufficiently high flow velocity the oscillation become chaotic. De Bedout et al [34] also came with a similar study using a magnetic transducer feedback controller to suppress flutter-type chaotic vibration in heat exchanger tubes. L.Wang and Q. Ni [35] studied the chaotic motion and Hopf bifurcation of a tubular cantilever beam, after following a similar procedure as reported in [33]. In this study they state that, the continuing chaotic vibration 
of the fixed-fixed beam may result in a support failure and can lead to the generation of a fixed-free boundary condition for the beam. They have also studied the effect of loose support location on the dynamics of the system.

\subsection{Problem definition}

In this thesis, we use the theory developed in $[33,35]$ to analyse FEI and post FEI nonlinear dynamics of heat exchanger tubes with multiple loose supports. As mentioned in [35], the chaotic and quasiperiodic vibration of the beam with fixed-fixed boundary conditions may leads to the generation of cantilever boundary conditions once the support fails. However, in [35] the influence of already existing loose supports (at the baffle at the centre of the fixed-fixed beam) on the dynamics of the tube has not been studied. Therefore in this thesis, we study the dynamics of the tube with two loose supports. First one is due to the transformation of one fixed joint to a loose support the end. The second one is due to the already existing loose supports at the mid span of the fixed-fixed beam. The response and the bifurcation of the system with respect to the free-gap, system parameters, fluid flow velocity will be studied.

\subsection{Thesis structure}

The entire thesis has been formatted in six chapters. The first chapter covers the introduction; here we mention the practical applications of a oscillating tube because of fluid flow, the mechanism of the instability due to fluid flow in heat exchanger, and a detailed review of instability and vibro-impact oscillation in cross flow heat exchanger tubes. Then we define the problem that we are going to focus on. In the second chapter different types of flow-induced forces on a flexible tube has been described. The main instabilities are due to drag and lift force on the cylinder because of the fluid flow and fluid forces induced by impulsive body motion. Also we explain the formulation for delayed displacement term in the system equation due to the flow retardation. The third chapter models the flexible tube subjected to cross flow as Euler-Bernoulli beam and discuss its numerical simulations. In the fourth chapter we discuss different approaches to to model an oscillating flexible tube with the baffle plate. In the last chapter we model the flexible tube with a cantilever boundary condition along with two independent baffles. Finally we conclude the entire thesis in chapter six. 


\section{Chapter 2}

\section{The flow-induced forces on a flexible tube}

In this chapter, section 2.1 explains the formatiulation of delayed displacement due to the flow retardation. Section 2.2 derives the fluid forces acting on the tube due to drag and lift. Section 2.3 mentions the fluid force induced by impulsive body motion. Finally the section 2.4 we arrive at the resultant fluid force on tube array along the lift-force direction. Figure 2.1 shows a typical tube bundle that we consider to derive the fluid forces. The motion of one cylindrical tube will influence the surrounding fluid motion and the disturbance in the fluid will excite the neighbouring cylindrical tube. For a double row cylindrical tube bundle (See Fig. 2.1), it has been assumed that the motion of the $i^{\text {th }}$ cylinder affects the motion of cylinder $i-1$ and cylinder $i+1$. Also the motion of cylinder $i-1$ produces a disturbance on the cylinder $i$ but not on the cylinder $i+1$, directly. But the resulting motion of the cylinder $i$ due to the cylinder $i-1$ produces a disturbance on the cylinder $i+1$. So $i-1$ has an indirect influence on $i+1$, therefore results in an indirect coupling effect [36].

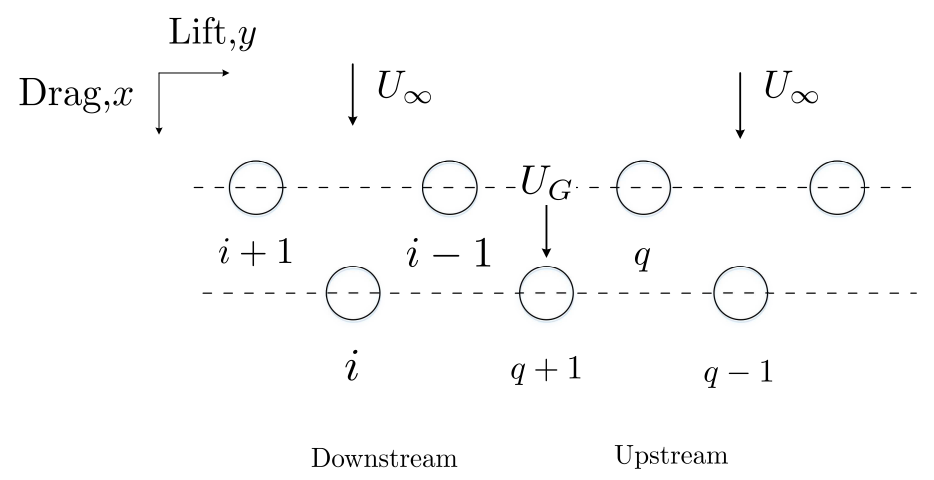

Figure 2.1: Schematic representation of double row cylindrical tube bundle. $U \infty$ is the free stream velocity, $U_{G}$ is the reference free-gap velocity. For an upstream cylinder $q$, approach velocity is $\infty$. 


\subsection{Flow retardation}

In a fluid flowing tube bundle, if there is a case where a small change in displacement leads to a large change in the fluid force coefficient, then the retardation of the flow approaching the tube has to be considered. In a two-dimensional flow, this retardation in the flow has been accounted by introducing a delay term for displacement as $x(t-\Delta t)$ and $y(t-\Delta t)$. The delayed term can be explained like this; the fluid impinge on the tube at time $t$ would have impinged on the cylinder if the velocity had been steady. So one can assume a steady velocity after introducing a delay to displacement terms as explained above. The quasi-steady theory of wake-induced flutter for a rigidly fixed wake-generating body had been discussed in [37], and obtained the expression for time delay. Later this method has been used in $[36,38]$ to analyse the retardation effect in cross flow tube arrays. Here, a brief derivation has been presented to obtain an expression for the time delay $\Delta t$ associated with the flow past cylinder. When a fluid flowing with a constant velocity $U$ impinging on a tube, then the time taken to travel the fluid from a distance $x=x_{1}$ to $x=R+\Delta R$ is given below:

$$
t=\frac{x_{1}-R-\Delta R}{U}
$$

where $R$ is the radius of the tube. When a fluid flow approaches near to the tube stagnation point, the velocity get retarded. Now if the approach velocity is taken as varying velocity, $U_{\text {apr }}$, then the time travel is calculated as follows;

$$
t+\Delta t=\int_{R+\Delta R}^{x} \frac{1}{U_{\mathrm{apr}}} d x
$$

Using potential-flow theory around an isolated cylinder, then $U_{\mathrm{apr}}=U\left(1-R^{2} / x^{2}\right)$, that gives;

$$
\Delta t=\frac{R}{2 U}\left[\ln \left(\frac{x_{1}-R}{x_{1}+R}\right)-\ln \left(\frac{\Delta R}{2 R+\Delta R}\right)\right] .
$$

Simpson and Flower suggested in [37], that $x_{1}$ is assumed as large when compared with $\Delta R$. Then we get the following expression for $\Delta t$ :

$$
\Delta t=\frac{R}{2 U} \ln \left(\frac{2 R}{\Delta R}\right)
$$

For a specific value of $\Delta R$, one may write the above expression as;

$$
\Delta t=\mu D / U
$$

According to [36] for a rotated triangular array $(P / D=1.375) \mu=1$. Where $D$ is the tube diameter and $P$ is the tube bundle pitch. In this thesis the same value for $\mu$ has been used.

\subsection{Drag and lift force on cylinder due to fluid flow}

The distribution of pressure around an object in a moving fluid is not uniform. This non-uniform pressure distribution leads to the generation of drag force along the flow direction and lift force 
along the transverse direction. With reference to the Fig 2.1, the force acting on the $i^{\text {th }}$ tube in drag direction is

$$
F_{x_{i}}=\frac{1}{2} \rho U_{\infty}^{2} L D\left[C_{D}\left(1-\frac{2}{U_{\infty} a}\right) \dot{x}+C_{L} \frac{1}{U_{\infty} a} \dot{y}\right],
$$

and the force acting on the $i^{\text {th }}$ cylinder in lift direction is

$$
F_{y_{i}}=\frac{1}{2} \rho U_{\infty}^{2} L D\left[C_{L}\left(1-\frac{2}{U_{\infty} a}\right) \dot{x}-C_{D} \frac{1}{U_{\infty} a} \dot{y}\right] .
$$

A detailed derivation of the above expression is given in [36]. Here, $\rho$ is the density of the flowing fluid with free stream velocity $U_{\infty}$ over the cylinder tube. $L$ and $D$ are the length and density of the cylinder respectively. $C_{D}$ and $C_{L}$ are the non-dimensional drag and lift force coefficients with respect to $U_{\infty}$. The term $a$ is the ratio of reference gap velocity $\left(U_{G}\right)$ to free stream velocity and is defined as $a=U_{G} / U_{\infty}$. We assume that $C_{L}$ can be expressed in linear form as follows;

$$
C_{L}=C_{L_{0}}+x \frac{\partial C_{L}}{\partial x}+y \frac{\partial C_{L}}{\partial y},
$$

similarly we expand $C_{D}$ as

$$
C_{D}=C_{D_{0}}+x \frac{\partial C_{D}}{\partial x}+y \frac{\partial C_{D}}{\partial y},
$$

here $x$ and $y$ are assumed as small displacements. Substituting the above expressions (Eq. (2.8) and Eq. (2.9)) in Eq. (2.7) and in Eq. (2.6) we obtain the drag force $F_{x}$ as follows:

$$
\begin{gathered}
F_{x}=\frac{1}{2} \rho U_{\infty}^{2} L D\left[C_{D_{0}}+x \frac{\partial C_{D}}{\partial x}+y \frac{\partial C_{D}}{\partial y}-\left(C_{D_{0}}+x \frac{\partial C_{D}}{\partial x}+y \frac{\partial C_{D}}{\partial y}\right) \frac{2}{U_{\infty} a} \dot{x}\right. \\
\left.+\left(C_{L_{0}}+x \frac{\partial C_{L}}{\partial x}+y \frac{\partial C_{L}}{\partial y}\right) \frac{\dot{y}}{U_{\infty} a}\right] .
\end{gathered}
$$

After neglecting $x \dot{x}$ and $y \dot{y}$ and introducing the delay in the displacement terms as in section 2.1, one can get the following expressions for the drag and lift forces

$$
\boldsymbol{F}_{i}=\frac{1}{2} \rho U_{\infty}^{2} L D\left(\frac{1}{U_{\infty} a} \mathbf{Q} \dot{\boldsymbol{x}}(t)+\mathbf{R} \boldsymbol{x}(t-\Delta t)+\boldsymbol{F}_{0}\right) .
$$

Here, $\boldsymbol{x}=[x, y]^{\mathrm{T}}, \boldsymbol{F}_{\boldsymbol{i}}=\left[F_{x_{i}}, F_{y_{i}}\right]^{\mathrm{T}}$ and

$$
\mathbf{Q}=\left[\begin{array}{cc}
-2 C_{D_{0}} & C_{L_{0}} \\
-2 C_{L_{0}} & -C_{D_{0}}
\end{array}\right], \quad \mathbf{R}=\left[\begin{array}{cc}
\frac{\partial C_{D}}{\partial x} & \frac{\partial C_{D}}{\partial y} \\
\frac{\partial C_{L}}{\partial x} & \frac{\partial C_{L}}{\partial y}
\end{array}\right], \text { and } \boldsymbol{F}_{0}=\left[\begin{array}{c}
C_{D_{0}} \\
C_{L_{0}}
\end{array}\right]
$$

\subsection{Fluid forces induced by impulsive body motion}

When a fluid flows around an object in a closed fluid domain, a force induced by the impulsive body motion will generate. This impulsive force was obtained after analysing the continuity and Navier-Stokes equations, and a force model for fluid force induced by an impulsive motion of a body subject to cross-flow has been developed in [39]. For a cylindrical body, the fluid force induced by 
impulsive body motion along the drag direction $(x)$ and lift direction $(y)$, is given as follows;

$$
\boldsymbol{F}_{1 a}=-\frac{\pi}{4} \rho L D^{2} \mathbf{C}_{m a} \ddot{\boldsymbol{x}}
$$

where $\mathbf{C}_{m a}$ is the added-mass coefficient matrix in a quiescent fluid.

\subsection{Resultant fluid forces acting on a tube in a tube array along the lift direction}

In the case of symmetrically positioned single flexible tube with respect to the adjacent cylinders, the following conditions hold

$$
C_{L 0}=\partial C_{L} / \partial x=\partial C_{D} / \partial y=0 .
$$

Generally the coupling terms in the added mass matrix $\mathbf{C}_{m a}$ are very small, so Eq. (2.11) and Eq. (2.13) can be combined in the decoupled form as follows

$$
\boldsymbol{F}_{i}=-\frac{\pi}{4} \rho D^{2} \mathbf{C}_{m a} \ddot{\boldsymbol{x}}+\frac{1}{2} \rho U_{\infty}^{2} L D\left(\frac{1}{U_{\infty} a} \mathbf{Q} \dot{\boldsymbol{x}}(t)+\mathbf{R} \boldsymbol{x}(t-\Delta t)+\boldsymbol{F}_{0}\right) .
$$

As we discussed in the introduction, for a cross flow, the instability is mainly because of lift forces and the contribution of drag force on instability is less. Since the forces along $x$ and $y$ direction are decoupled, the force along the lift direction can be written as follows

$$
F_{y_{i}}=-\frac{\pi}{4} \rho L D^{2} C_{m a} \ddot{y}-\frac{1}{2 a} \rho U_{\infty} L D C_{D_{0}} \dot{y}(t)+\frac{1}{2} \rho U_{\infty}^{2} L D \frac{\partial C_{L}}{\partial y} y(t-\Delta t) .
$$

In an upstream-cylinder $a$ must be unity, because the approach velocity is $U_{\infty}$. Therefore, for cylinder $q$, we take $U_{\infty}=U_{G}=U$. Hence, the lift force $F$ acting on a flexible tube, surrounded by several rigid tubes, under cross flow can be written as follows;

$$
F_{q}=-\frac{\pi}{4} \rho D^{2} C_{m a} \ddot{y}-\frac{1}{2} \rho U D C_{D_{0}} \dot{y}(t)+\frac{1}{2} \rho U^{2} D \frac{\partial C_{L}}{\partial y} y(t-\Delta t) .
$$

If we take $q$ as flexible tube surrounded by many rigid tubes, the above force (Eq. (2.17)) can be used to represent fluid forces acting on a flexible tube. In the next chapter we use Euler-Bernouli beam equation for representing the dynamics of the tubes. Later we introduce fluid forced developed in this chapter on the beam model for predicting FEI. 


\section{Chapter 3}

\section{Modelling of a flexible tube subjected to cross flow}

In this chapter, a dynamic model of heat exchanger tube has been developed. It is assumed that in a cross flow heat exchanger, a single flexible tube is surrounded by many rigid tubes. When we consider only first mode of beam vibration, one can model the system as a single degree of freedom mass-spring-damper (for one dimensional analysis) or two degree of freedom mass-spring-damper (for two dimensional analysis) with external excitations. Now, if we take more modes, the system has to be modelled as Euler-Bernoulli beam with external excitation. For a long tube, the high amplitude oscillation is restricted by placing many supports along the span at different locations, known as baffles. As we discussed in section 1.1, the diametrical expansion the of tube is allowed by making gaps at tube-baffle contact. And this gap allows the tube to oscillate in some extent, that leads to chaotic oscillation.

\subsection{The equation of motion of the beam}

With reference to Fig. 3.1, the equation of motion of Euler-Bernoulli beam [33, 35] is given below (see Eq. (3.1))

$$
E I \frac{\partial^{4} w}{\partial x^{4}}+c \frac{\partial w}{\partial t}+m \frac{\partial^{2} w}{\partial t^{2}}+\delta\left(x-x_{b}\right) f(w)=F(w, \dot{w}, \ddot{w}) .
$$

Where $w=w(x, t)$ is the lateral displacement of the tube in perpendicular direction of the fluid flow. $E$ is the Young's modulus, $I$ is the second moment of area, and $c$ is the beam structural damping coefficient. The mass per unit length of the beam including the flowing fluid mass through the tube is given as $m$. For a constant fluid flow with a velocity $U$, the mass $m$ is also constant.

A baffle has been kept at a distance $x=x_{b}$ as shown in Fig. 3.1(b). In realty the baffle is a rigid support, but in this study a spring with high stiffness has been used to model it. In this thesis two baffle models has been studied. The first one is a cubic spring model and the second one is tri-linear spring model. The support force because of baffle is defined as $\delta\left(x-x_{b}\right) f(w)$, where $\delta$ is the Diracs delta function. The RHS term $F(w, \dot{w}, \ddot{w})$ is the external force acting on the tube due to the fluid flow and has been derived in section 2.4. Here $y=w(x, t)$ and $F=F(w, \dot{w}, \ddot{w})$. On substituting 


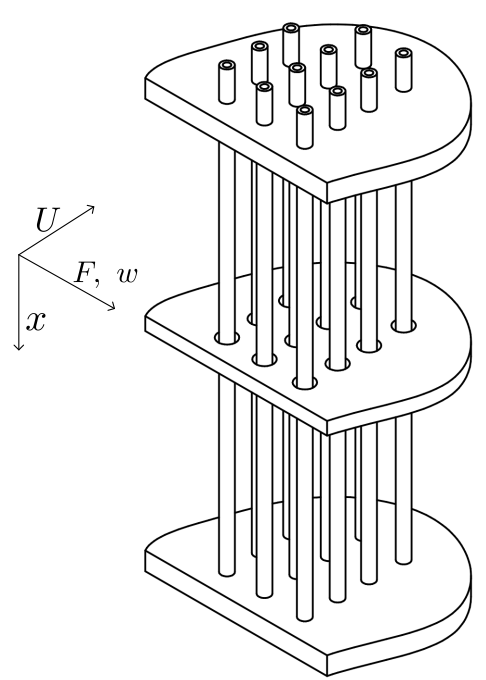

(a)

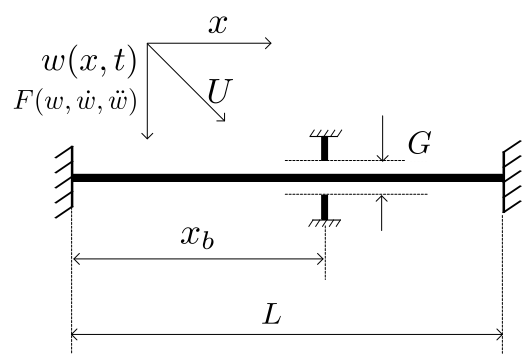

$(b)$

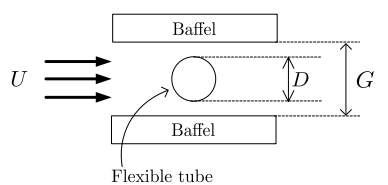

(c)

Figure 3.1: (a) shows a heat exchanger with single baffle at the centre. (b) shows a schematic representation of a single tube as fixed-fixed beam modelling. (c) shows the side view of diagram (b) at baffle cross section. Note that figures are not in same scale

$F(w, \dot{w}, \ddot{w})$ from Eq. (2.17) in Eq. (3.1), we get;

$$
E I \frac{\partial^{4} w}{\partial x^{4}}+(c-B) \frac{\partial w}{\partial t}+(m-M) \frac{\partial^{2} w}{\partial t^{2}}-C w(x, t-\Delta t)+\delta\left(x-x_{b}\right) f(w)=0 .
$$

where

$$
M=-\frac{\pi}{4} \rho D^{2} C_{m a}, \quad B=-\frac{1}{2} \rho U D C_{D}, \text { and } C=\frac{1}{2} \rho U^{2} D \frac{\partial C_{L}}{\partial w}
$$

\subsection{Non-dimensionalisation}

The non-dimensionalisation removes the units of physical quantities partially or fully by a suitable substitution. We introduce the following non dimensional variables

$$
\eta=\frac{w}{D}, \xi=\frac{x}{L}, \tau=\Omega_{1} t
$$

Where $L$ and $D$ are the length and diameter of the tube respectively. For more clarity in the derivation, first let us non-dimensionalise Eq. (3.2) except for the last two terms as follows:

$$
E I \frac{D}{L^{4}} \frac{\partial^{4} \eta}{\partial \xi^{4}}+\left(c+\frac{1}{2} \rho U D C_{D}\right) \Omega_{1} D \frac{\partial \eta}{\partial \tau}+\left(m+\frac{\pi}{4} \rho D^{2} C_{m a}\right) D \Omega_{1}^{2} \frac{\partial^{2} \eta}{\partial \tau^{2}}
$$

substitute $\zeta=\frac{c}{\Omega_{1} m}$ in the above equation (Eq. (3.5)), the second term becomes

$$
\left(c+\frac{1}{2} \rho U D C_{D}\right) \Omega_{1} D \frac{\partial \eta}{\partial \tau}=\Omega_{1}^{2} \rho D^{3} \tilde{m}\left(\zeta+\frac{\tilde{U} C_{D}}{4 \pi \tilde{m}}\right) \frac{\partial \eta}{\partial \tau}
$$


where $\tilde{m}=\frac{m}{\rho D^{2}}$ and $\tilde{U}=\frac{2 \pi U}{D \Omega_{1}}$, the third term of Eq. (3.5) becomes

$$
\left(m+\frac{\pi}{4} \rho D^{2} C_{m a}\right) D \Omega_{1}^{2} \frac{\partial^{2} \eta}{\partial \tau^{2}}=\Omega_{1}^{2} \rho D^{3} \tilde{m}\left(1+\frac{1}{\tilde{m}} \frac{\pi}{4} C_{m a}\right) \frac{\partial^{2} \eta}{\partial \tau^{2}}
$$

substitute $\frac{1}{1-\beta}=1+\frac{1}{\tilde{m}} \frac{\pi}{4} C_{m a}$ in the above equation and finally Eq. (3.5) can be rewritten as

$$
\Omega_{1}^{2} \rho D^{3} \tilde{m}\left(\frac{1}{1-\beta} \frac{\partial^{2} \eta}{\partial \tau^{2}}+\left(\zeta+\frac{\tilde{U} C_{D}}{4 \pi \tilde{m}}\right) \frac{\partial \eta}{\partial \tau}+\frac{1}{\lambda_{1}^{4}}\left(\frac{\partial^{4} \eta}{\partial \xi^{4}}\right)\right)
$$

where $\lambda_{1}^{2}=\Omega_{1} \sqrt{\frac{m L^{4}}{E I}}$, and $\lambda_{1}$ is the dimensionless eigenvalue of the first mode for a beam. From section 2.1, it has been found that the delay time $\Delta t=D / U$ for $\mu=1$. Introduce a term $T=2 \pi / \tilde{U}$, then the delay time $\Delta t$ becomes $\Delta t=\frac{T}{\Omega_{1}}$. Hence the delayed displacement of the Eq. (3.2) can be written as follow

$$
w(x, t-\Delta t)=w\left(x, t-\frac{T}{\Omega_{1}}\right)=w\left(\xi L, \frac{\tau-T}{\Omega_{1}}\right)=D \eta(\xi, \tau-T)
$$

So the fourth term of Eq. (3.2) becomes;

$$
\frac{1}{2} \rho U^{2} D \frac{\partial C_{L}}{\partial \eta D} w(x, t-\Delta t)=\rho \Omega_{1}^{2} D^{3} \tilde{m}\left(\frac{\tilde{U}^{2}}{8 \pi^{2} \tilde{m}} \frac{\partial C_{L}}{\partial \eta}\right) \eta(\xi, \tau-T)
$$

and finally the last term of the Eq. (3.2) can be written as

$$
\delta\left(x-x_{b}\right) f(w)=\delta\left(\xi L-\xi_{b} L\right) f(\eta D)=D \delta\left(\xi-\xi_{b}\right) f(\eta)
$$

or

$$
D \delta\left(\xi-\xi_{b}\right) f(\eta)=\tilde{m} \rho \Omega_{1}^{2} D^{3} \delta\left(\xi-\xi_{b}\right) \tilde{f}(\eta)
$$

where $\tilde{f}(\eta)=\frac{f}{m \Omega_{1}^{2}}$. So after considering all the above derivations, Eq. (3.2) can be written as follows

$$
\begin{gathered}
\frac{1}{1-\beta} \frac{\partial^{2} \eta}{\partial \tau^{2}}(\xi, \tau)+\left(\zeta+\frac{\tilde{U} C_{D}}{4 \pi \tilde{m}}\right) \frac{\partial \eta}{\partial \tau}(\xi, \tau)+\frac{1}{\lambda_{1}^{4}}\left(\frac{\partial^{4} \eta}{\partial \xi^{4}}(\xi, \tau)\right) \\
-\left(\frac{\tilde{U}^{2}}{8 \pi^{2} \tilde{m}}\right) \frac{\partial C_{L}}{\partial \eta} \eta(\xi, \tau-T)+\delta\left(\xi-\xi_{b}\right) \tilde{f}(\eta)=0 .
\end{gathered}
$$

Finally we obtain a non-dimensional partial differential equation (Eq. (3.13)) in $\eta$ and $\tau$. In the next section the partial differential equation will be descritized to get a system of first order delay differential equations using Galerkin method. 


\subsection{Discretization using Galerkin approximation}

For a beam, the contribution of higher modes in the response is negligible when compare with lower modes. Therefore, we assume a series solution with $N$ number of terms as shown below

$$
\eta(\xi, \tau)=\sum_{i=1}^{N} \phi_{i}(\xi) q_{i}(\tau)=\phi^{\mathrm{T}}(\xi) \boldsymbol{q}(\tau)
$$

Where $\boldsymbol{\phi}=\left[\phi_{1}, \phi_{2}, \ldots, \phi_{N}\right]^{\mathrm{T}}$ denotes the shape function of beam and they depend on the boundary condition of the beam. The generalized coordinates are $\boldsymbol{q}=\left[q_{1}, q_{2}, \ldots, q_{N}\right]^{\mathrm{T}}$. Since the normalized length of beam $\eta$ is varying from 0 to 1 , a domain $\eta \in[0,1]$ has been selected. Now substitute the above series solution into the Eq. (3.13), and pre-multiply the resulting expression with $\phi(\xi)$; then integrating over the domain $\eta \in[0,1]$, we get

$$
\begin{gathered}
\frac{1}{1-\beta} \int_{0}^{1} \phi \phi^{\mathrm{T}} d \xi \ddot{\boldsymbol{q}}(\tau)+\left(\zeta+\frac{\tilde{U} C_{D}}{4 \pi \tilde{m}}\right) \int_{0}^{1} \phi \phi^{\mathrm{T}} d \xi \dot{\boldsymbol{q}}(\tau)+\frac{1}{\lambda_{1}^{4}} \int_{0}^{1} \phi\left(\phi^{i v}\right)^{\mathrm{T}} d \xi \boldsymbol{q}(\tau) \\
-\left(\frac{\tilde{U}^{2}}{8 \pi^{2} \tilde{m}}\right) \frac{\partial C_{L}}{\partial \eta} \int_{0}^{1} \phi \phi^{\mathrm{T}} d \xi \boldsymbol{q}(\tau-T)+\int_{0}^{1} \phi \delta\left(\xi-\xi_{b}\right) \tilde{f}(\eta) d \xi=0
\end{gathered}
$$

Where $\phi=\phi(\xi)$ and $\phi^{i v}=\frac{\partial^{4}}{\partial \xi^{4}} \phi$. The standard shape function of beam $\phi(\xi)$ are the dimensionless ortho-normal eigenfunctions. And any orthonormal shape-function has the following properties

$$
\begin{gathered}
\int_{0}^{1} \phi(\xi) \phi^{T}(\xi) d \xi= \begin{cases}1 & \text { for } i=j \\
0 & \text { for } i \neq j\end{cases} \\
\int_{0}^{1} \phi(\xi) \frac{\partial^{4}}{\partial \xi^{4}} \phi^{T}(\xi) d \xi=\left\{\begin{array}{cc}
\lambda_{i}^{4} & \text { for } i=j \\
0 & \text { for } i \neq j
\end{array}\right.
\end{gathered}
$$

and for Dirac $\delta$ function one can arrive the following expression

$$
\int_{0}^{1} \phi(\xi) \delta\left(\xi-\xi_{b}\right) \tilde{f}(\eta) d \xi=\left\{\begin{array}{cc}
\phi_{i}\left(\xi_{b}\right) \tilde{f}\left(\eta_{b}\right) & \text { for } i=j \\
0 & \text { for } i \neq j
\end{array}\right.
$$

so Eq. (3.15) becomes

$$
\begin{gathered}
\frac{1}{1-\beta} \ddot{q}_{i}(\tau)+\left(\zeta+\frac{\tilde{U} C_{D}}{4 \pi \tilde{m}}\right) \dot{q}_{i}(\tau)+\left(\frac{\lambda_{i}}{\lambda_{1}}\right)^{2} q_{i}(\tau) \\
-\frac{\tilde{U}^{2}}{8 \pi^{2} \tilde{m}} \frac{\partial C_{L}}{\partial \eta} q_{i}(\tau-T)+\phi_{i}\left(\xi_{b}\right) \tilde{f}\left(\eta_{b}\right)=0, i=1,2, \ldots, N
\end{gathered}
$$

\subsection{Generalized equation of motion in first-order form}

The equation of motion (Eq. (3.19)) can be written in the state space form as shown below

$$
\dot{\boldsymbol{z}}=\mathbf{A} \boldsymbol{z}+\mathbf{B} \boldsymbol{z}(\tau-T)+\boldsymbol{F} .
$$


Where $\boldsymbol{z}=\left[\boldsymbol{q}^{\mathrm{T}}, \dot{\boldsymbol{q}}^{\mathrm{T}}\right]^{\mathrm{T}}$ is the state vector. The above first order state space equations (Eq. (3.20)) has been obtained after making the following substitutions in Eq. (3.19)

$$
\begin{gathered}
a=\frac{1}{1-\beta}, b_{i}=\frac{\delta_{i} v_{i}}{\pi}+\frac{\tilde{U} C_{D}}{4 \pi \tilde{m}}, \text { where } v_{i}=\frac{\lambda_{i}}{\lambda_{1}}, \\
c_{i}=v_{i}^{2}, \text { and } d=\frac{\tilde{U}^{2}}{8 \pi^{2} \tilde{m}} \frac{\partial C_{L}}{\partial \eta}
\end{gathered}
$$

here $v_{i}$ is the ratio of non-dimensional natural frequencies to the first non-dimensional natural frequency. For small damping, the damping term is replaced by modal damping $\delta_{i} v_{i} / \pi$. The matrices $\mathbf{A}$ and $\mathbf{B}$ are defined as follows:

$$
\mathbf{A}=\left[\begin{array}{ll}
\mathbf{0} & \mathbf{I} \\
\mathbf{c} & \mathbf{b}
\end{array}\right]_{2 N \times 2 N} \text { and } \mathbf{B}=\frac{d}{a}\left[\begin{array}{ll}
\mathbf{0} & \mathbf{0} \\
\mathbf{I} & \mathbf{0}
\end{array}\right]_{2 N \times 2 N}
$$

where

$$
\mathbf{b}=-\frac{1}{a}\left[\begin{array}{cccc}
b_{1} & 0 & \cdots & 0 \\
0 & b_{2} & \cdots & 0 \\
\vdots & \vdots & \ddots & \vdots \\
0 & 0 & \cdots & b_{N}
\end{array}\right]_{N \times N} \text { and } \mathbf{c}=-\frac{1}{a}\left[\begin{array}{cccc}
c_{1} & 0 & \cdots & 0 \\
0 & c_{2} & \cdots & 0 \\
\vdots & \vdots & \ddots & \vdots \\
0 & 0 & \cdots & c_{N}
\end{array}\right]_{N \times N}
$$

and $\mathbf{I}$ is the identity matrix of the size $N \times N$. The force vector $\boldsymbol{F}$ has to come from baffle type and node on which the baffle has been fixed. In the coming chapters we will discuss about $\boldsymbol{F}$ in detail.

\subsection{Numerical solution}

In the previous section we have arrived at $2 N$ first order delay differential equations (see Eq. (3.20)). Solution of these equations can be obtained by integrating numerically with the help of fourthorder Runge-Kutta method with appropriate time step. Since Matlab has its own delay differential equation solver "dde23" we use the same solver for finding the solutions.

In the upcoming chapters we will demonstrate three different modelling of the flexible heat exchanger tube by considering appropriate boundary conditions. Since we have already non dimensionalised all the physical quantities, we remove the 'tildes' on the symbols from now on for clarity. 


\section{Chapter 4}

\section{Fixed-fixed beam modelling}

In this chapter, we follow the same modelling as discussed by Païdoussis [33]. The flexible tube is modelled as a fixed-fixed beam (see Fig. 4.1) with a baffle. In section 4.1, we define the the shape functions $\left(\phi_{i}(\xi)\right)$ for a fixed fixed beam. In section 4.2, we discuss about Hopf bifurcation and critical flow velocities of the system. Finally in sections 4.4 and 4.5 we show the results from numerical simulation of the system.

\subsection{Shape functions}

For a unit length fixed-fixed beam, the shape function $\phi(\xi)$ (see Eq. (3.14)) are given by

$$
\phi_{i}(\xi)=\left(\cosh \lambda_{i} \xi-\cos \lambda_{i} \xi\right)-\sigma_{i}\left(\sinh \lambda_{i} \xi-\sin \lambda_{i} \xi\right)
$$

where

$$
\sigma_{i}=\frac{\cos \lambda_{i}-\cosh \lambda_{i}}{\sin \lambda_{i}-\sinh \lambda_{i}} .
$$

It has been found that the effect of the higher modes on the magnitude of vibration is negligible. So we use only five modes for the entire simulations. The dimensionless eigenvalue $\lambda_{i}$ is given below

$$
\boldsymbol{\lambda}=[4.7300,7.8532,10.9956,14.1372,17.2788]
$$

\subsection{Hopf bifurcation}

When a system losses its stable equilibrium and goes to an amplified steady oscillatory state, it is knows as Hopf bifurcation. The non-dimensional velocity at which the Hopf bifurcation of the system (Eq. (3.19)) occurs can be found out by the following method. After keeping all parameters constant, increases the non-dimensional velocity $U$ gradually from zero until the system losses its stability. This velocity at which the system losses stability is know as critical velocity $\left(U_{H}\right)$. Thereafter, for any velocity grater than critical velocity, the systems amplitude starts to increase. But for a nonlinear system, the nonlinear forces counter-balance the further amplification and it reaches a steady amplitude. 


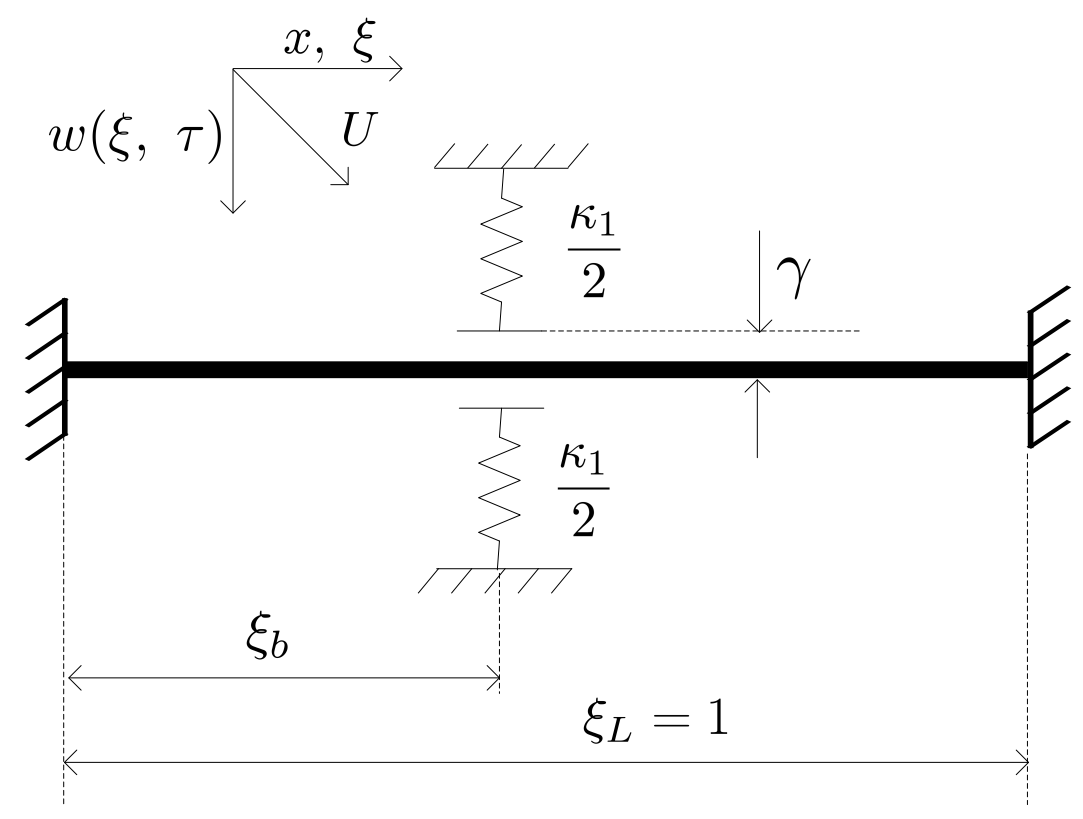

Figure 4.1: The schematic representation of a fixed-fixed beam with mid-span loose support.

For the entire thesis, unless otherwise mentioned, we use the following parameters

$$
C_{D}=0.26, \frac{\partial C_{L}}{\partial \eta}=-8.1, \tilde{m}=3, \delta_{i}=0.06, \beta=0.24
$$

Substituting the above parameters in Eq. (3.19) and then dropping the forcing term $\tilde{f}\left(\eta_{b}\right)$, we obtain the following equation

$$
\ddot{q}_{i}(\tau)+\left(\beta_{1}+\beta_{2} U\right) \dot{q}_{i}(\tau)+\beta_{3} q_{i}(\tau)+\beta_{4} U^{2} q_{i}(\tau-T)=0
$$

where $\beta_{1}=0.0145 v_{i}, \beta_{2}=0.00524, \beta_{3}=0.76 v_{i}^{2}$, and $\beta_{4}=0.026$. Since the above equations are decoupled for any number of modes $N$, an independent solution can be assumed as follows; for $i^{\text {th }}$ mode:

$$
q_{i}=q_{i_{0}} e^{j \omega_{i} \tau}
$$

where $j=\sqrt{-1}$ and $\omega_{i}$ is the dimensionless natural frequency. Now substitute the assumed solution into the Eq. (4.5) to get the following expressions:

$$
-\omega_{i}^{2}+\left(\beta_{1}+\beta_{2} U\right) j \omega_{i}+\left(\beta_{3}+\beta_{4} U^{2} e^{-j \omega_{i} T}\right)=0
$$

At Hopf bifurcation (the flow velocity become critical velocity, $U=U_{H}$ ) and $\omega_{i}$ should be purely real, and this gives the follows equations:

$$
-\omega_{i}^{2}+\beta_{3}+\beta_{4} U_{H}^{2} \cos \omega_{i} T=0, \text { and }\left(\beta_{1}+\beta_{2} U_{H}\right) \omega_{i}-\beta_{4} U_{H}^{2} \sin \omega_{i} T=0
$$

On solving Eq. (4.8) for $U_{H}$ and $\omega_{i}$ we get the critical velocity corresponds to different mode shapes as shown in the table 4.1. Table 4.1 shows the critical dimensionless flow velocity along with non-dimensional natural frequency for a fixed-fixed beam. It has been found that after the the fist 


\begin{tabular}{lll}
\hline Modes & $U_{H}$ & $\omega_{i}$ \\
\hline 1 & 1.785 & 0.824 \\
2 & 4.920 & 2.272 \\
3 & 9.645 & 4.455 \\
4 & 19.44 & 7.364 \\
5 & 23.818 & 11.00 \\
\hline
\end{tabular}

Table 4.1: Critical dimensionless flow velocity with non-dimensional natural frequency for a fixedfixed beam corresponds to the mode shape.

critical velocity $\left(U_{H} 1.785\right)$, the amplitude of the system increases. In our case because of the impact forces, the decoupled system (Eq. (4.5)) become coupled. So it is not possible to treat the system as a set of independent equations, hence critical velocity also changes. Results related to coupled systems are presented in the next sections.

\subsection{Constrained force modelling}

The restrained force $\tilde{f}\left(\eta_{b}\right)$ (in the system (Eq. 3.19)) because of the impact of the tube on the baffle has to be modelled mathematically. Ivanov [40] has proposed an analytical model to study the response of a vibro-impact systems. His method can be used to analysis the vibration of beam with a rigid stopper along a single transverse direction of the beam. Since in our case the baffle has to be kept in such a way that, a restricted oscillation of the beam should be allowed in both directions. So the model proposed by Ivanov [40] cannot be applied to baffle type stoppers. Inorder to overcome the above difficulty, Païdoussis [33] has used a spring model with high stiffness. He mainly considered two different types of springs, first one is a cubic spring and other one is a tri-linear spring model. In section 4.4, the bifurcation and the time response of the system has been shown for the cubic spring baffle. Likewise in section 4.5, bifurcation and the time response of the system for the tri-linear spring baffle are also shown.

\subsection{Modelling of baffle as a cubic spring}

As we discussed in the previous section, we consider a cubic spring model to represent a baffle. The equation of a cubic spring with a spring stiffness $\kappa$ is defined as follows

$$
\tilde{f}\left(\eta_{b}\right)=\kappa \eta_{b}^{3}
$$

The variation of the force with respect to deflection of the cubic-spring (for $\kappa=1$ ) is shown in Fig. 4.2. It can be observed that for a small deflection between $[-g, g]$, the spring restrained force is negligible. But, when the deflection increases beyond a certain region $([-g, g])$, the the spring shows a sharp increase in the restrained force. So when we model the baffle as cubic spring, the region $[-g, g]$ can be considered as the gap between the baffle and the tube. A further increase in the tube osculations will be arrested by the high restrained force of the cubic spring, to some extent. For the entire thesis work, we select $\kappa=1000$.

Substitute the the series solution (Eq. (3.14)) to the forcing term (Eq. (4.9)), the forcing vector 




Figure 4.2: The variation of cubic spring restrained force $\left(\tilde{f}\left(\eta_{b}\right)\right)$ with respect to the deflection $\eta$ for $\kappa=1$

in the Eq. (3.20) will become

$$
\boldsymbol{F}=\left[\boldsymbol{F}_{1}^{T}, \boldsymbol{F}_{2}^{T}\right]^{T}
$$

Where $\boldsymbol{F}_{1}$ is a zero vector of size $N \times 1$ and $\boldsymbol{F}_{2}=\kappa\left(\boldsymbol{\phi}^{T} \boldsymbol{q}\right)^{3} \phi$. We substitute this force vector $\boldsymbol{F}$ into the system equation (Eq. (3.20)) and integrate with Matlab ddesolver "dde23" with initial condition $\eta\left(\xi_{b}, t\right)=0.000001$ and $\frac{\partial}{\partial t} \eta\left(\xi_{b}, t\right)=0$.

\subsubsection{Bifurcation due to symmetric loading}

In this section we study the bifurcation and time response of the system with a cubic spring placed at the mid-span $\left(\xi_{b}=0.5\right)$ of the beam. Fig. 4.3 shows a bifurcation diagram of the system with respect to the dimensionless velocity $U$. From Fig. 4.3 it is clear that the system is stable for any velocity $U$ less than 1.78 . However, at $U=1.78$ the Hopf bifurcation occurs, thereafter the system start to lose its stability which leads to the birth of limit cycle. It has been observed that the system show an unstable response for a velocity range of $U=1.78$ to $U=2.1$. However, when we increase the velocity further, the amplitude of oscillation start to grow and maintain an amplified steady state until $U=3.6$. At this stage the system shows two steady extreme displacements. On a further increase of the velocity beyond $U=3.6$, it again starts to bifurcate (first post Hopf-bifurcation) and maintain a steady state with six different extreme displacements. A second post-Hopf bifurcation occur at $U=4.35$, where the system shows eight amplified steady extremes. It is clear from the figure that, a further increase of $U$ beyond $U=4.6$, the response of the system become highly chaotic. In order to get a clear picture of the behaviour of the system, we plot phase diagrams and time responses corresponds to the dimensionless velocity, $U$, as shown in Fig. 4.4. Fig. 4.4(a)-(b) shows the phase plot and the time response at a velocity $U=3$. It is clear from the response curve (Fig. 4.4(b)) that, the amplitude of oscillation was very less for a particular range of time. Further increase of time leads to an unstable state but later it will settle to an amplified steady state, at this stage (as we discussed in the previous paragraph) the phase diagram ( Fig. 4.4(a)) gives a limit 


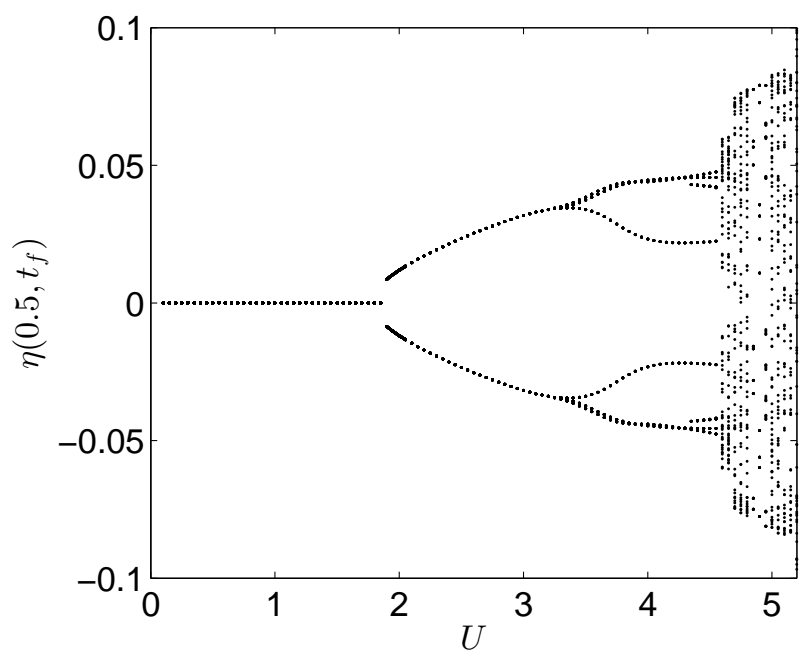

Figure 4.3: Bifurcation diagram of a fixed-fixed beam with cubic spring baffle located at $\xi=1 / 2$.

cycle. This time response and phase diagram indicate that that the tube is impacting the loose supports on both sides.

We have already seen from the bifurcation plot that a post-Hopf bifurcation starts at $U=3.6$ and is also evident in Figs. 4.4(c)-(d). At $U=3.6$, after leaving the first spring and before hitting the opposite spring, the tube oscillate locally one more time. That is why the phase plot have one rotation before changing the displacement sign. When we increase the velocity $U$ further, the response of the system becoming more and more complex. But some local steady state solutions were observed, the response is not chaotic when we take the velocities in between $U=4.85$ and $U=4.95$. The period of the oscillations are clearly visible from each response curve. For a chaotic system, since the number of possible amplitude of oscillations are more it is very difficult to extract the period of the response.

\subsubsection{Bifurcation due to asymmetric loading}

In the previous section, we were discussing about the symmetric loading, where we kept a cubic spring at the mid-span of the tube and measure the response at the $\xi=0.5$. Now in this section we will briefly study the effect of an asymmetric loading after keeping the baffle at $\xi_{b}=0.45$. Figure. 4.6 shows the bifurcation for unsymmetrical loose support (cubic spring). The Hopf bifurcation starts at $U=1.75$, where the system shows an amplified steady state after a particular time. As explained for symmetric loose support, here also tubes oscillate locally before going to the opposite side. It can be seen that a first post-Hopf bifurcation occurs at a dimensionless flow velocity $U=3.35$. Second post-Hopf bifurcation starts at $U=4.1$ and after $U=4.05$ the system become chaotic. Up to $U=4.3$. it can be seen that the system oscillate with a finite amplitude. But beyond $U=4.3$, the solution starts to blowup and becomes unstable.

For more clarity we analyse the phase plot and time response, as shown in Fig. 4.8. Only a small portion of the time response corresponds to $U=4.05$ has been shown. At $U=4.65$, the response starts to blow up. And a further increase in the velocity gives highly unstable response. Even though it gives some high response after $U=4.65$, no periodic solution was obtained, so in these 


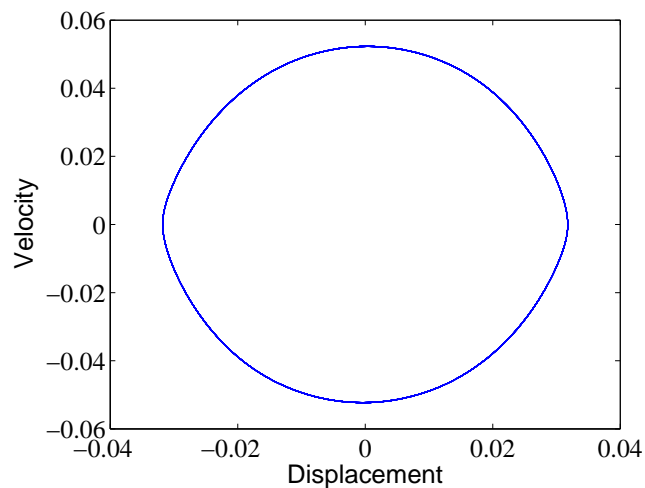

(a)

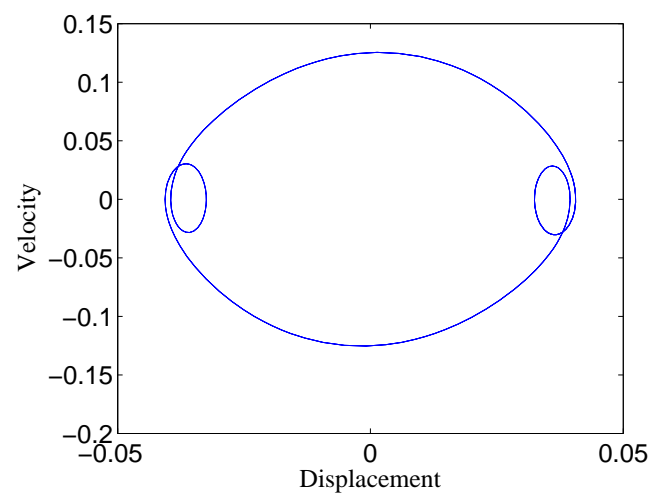

(c)

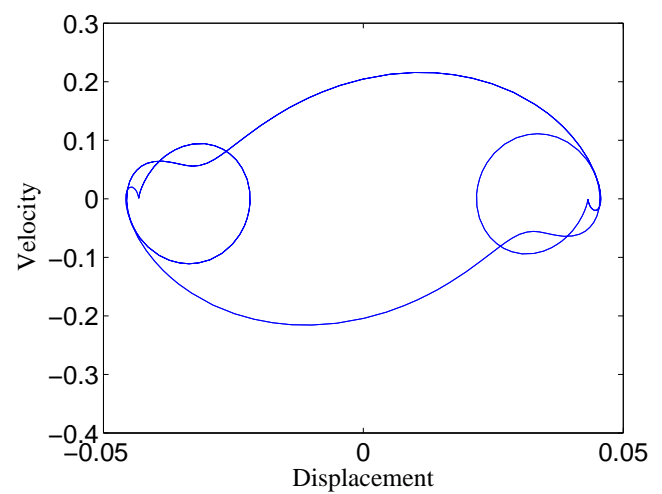

(e)

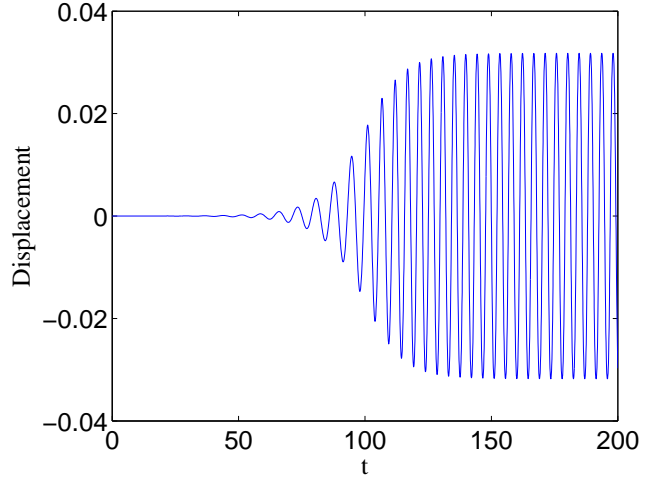

(b)

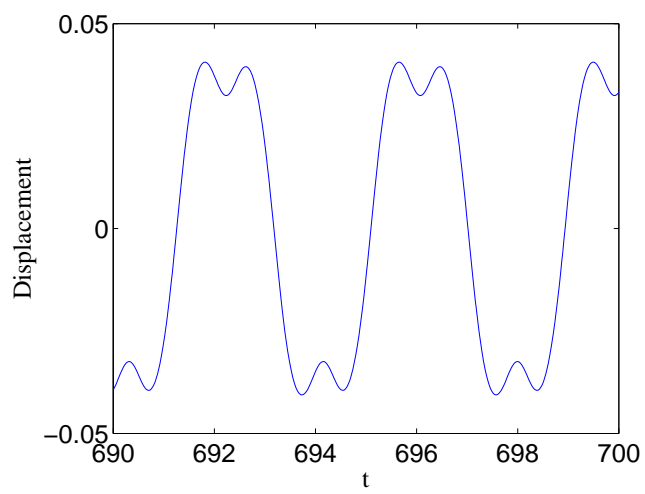

(d)

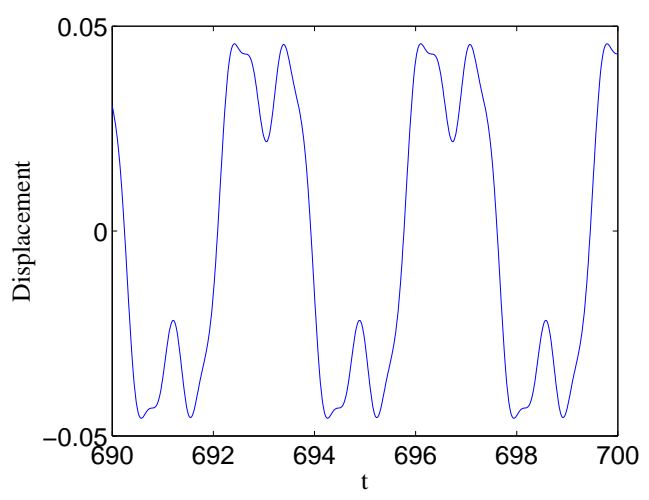

(f)

Figure 4.4: Phase plots and time responses associated with the bifurcation diagram of Fig. 4.3: (a) and (b) are for $U=3$; (c) and (d) are for $U=3.6$; (e) and (f) are for $U=4$; and (g) and (h) are for $U=4.3$. 


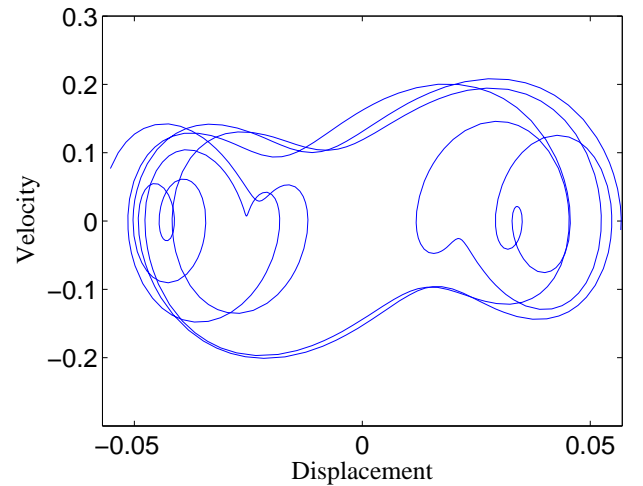

(a)

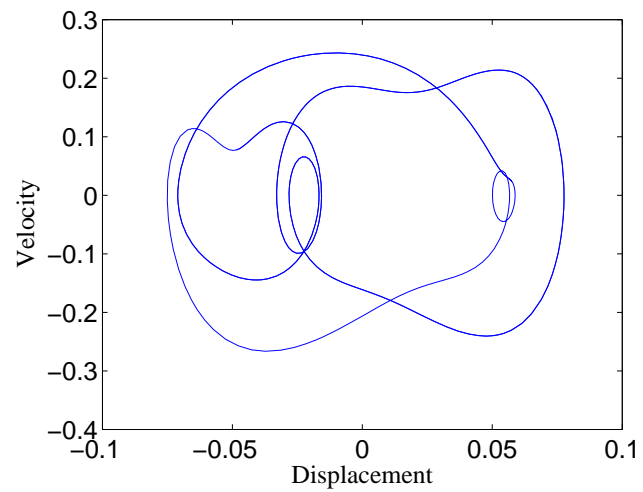

(c)

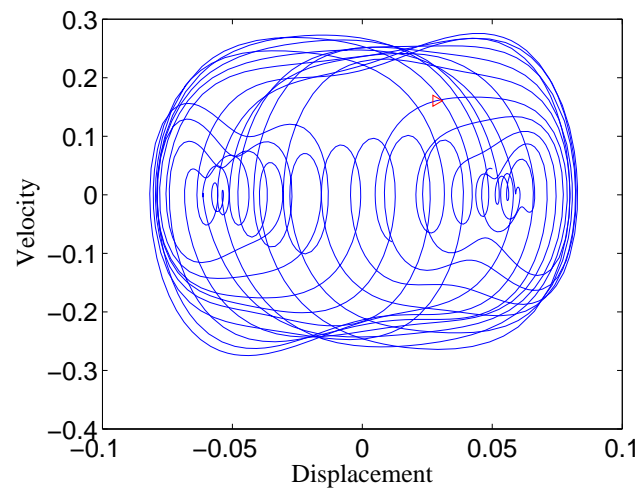

(e)

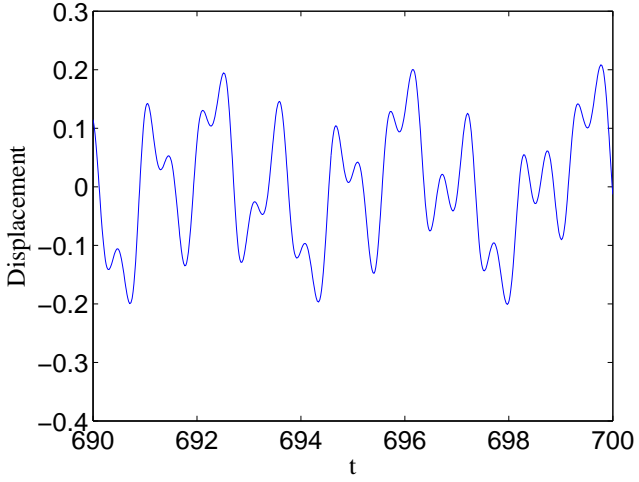

(b)

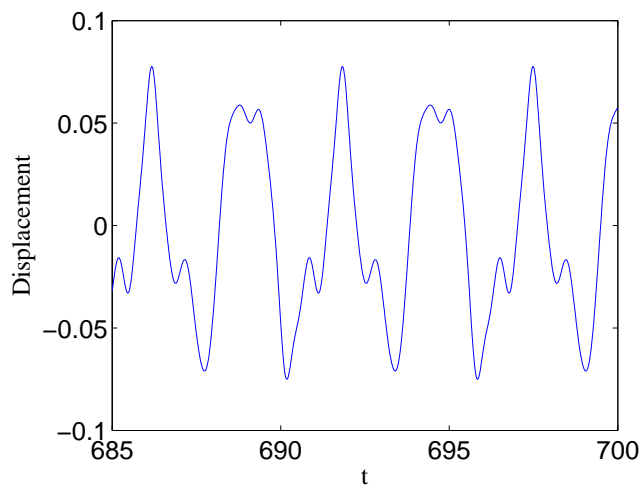

(d)

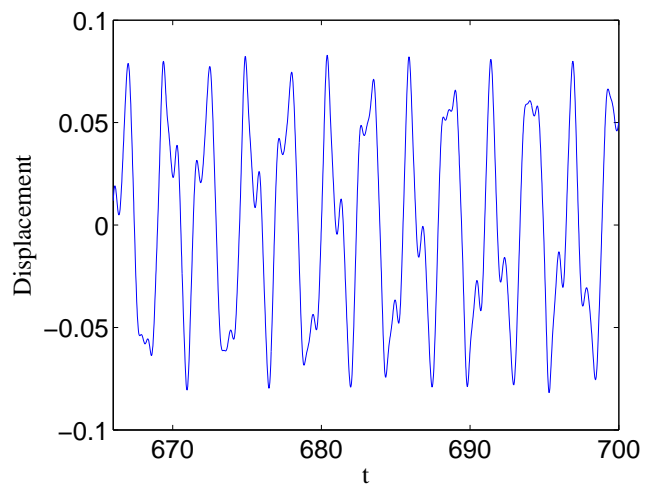

(f)

Figure 4.5: (a) Phase plots and time responses associated with the bifurcation diagram of Fig. 4.3, corresponds to U: (a) and (b) are for $U=4.65$; (c) and (d) are for $U=4.85$; and (e) and (f) are for $U=5$. 


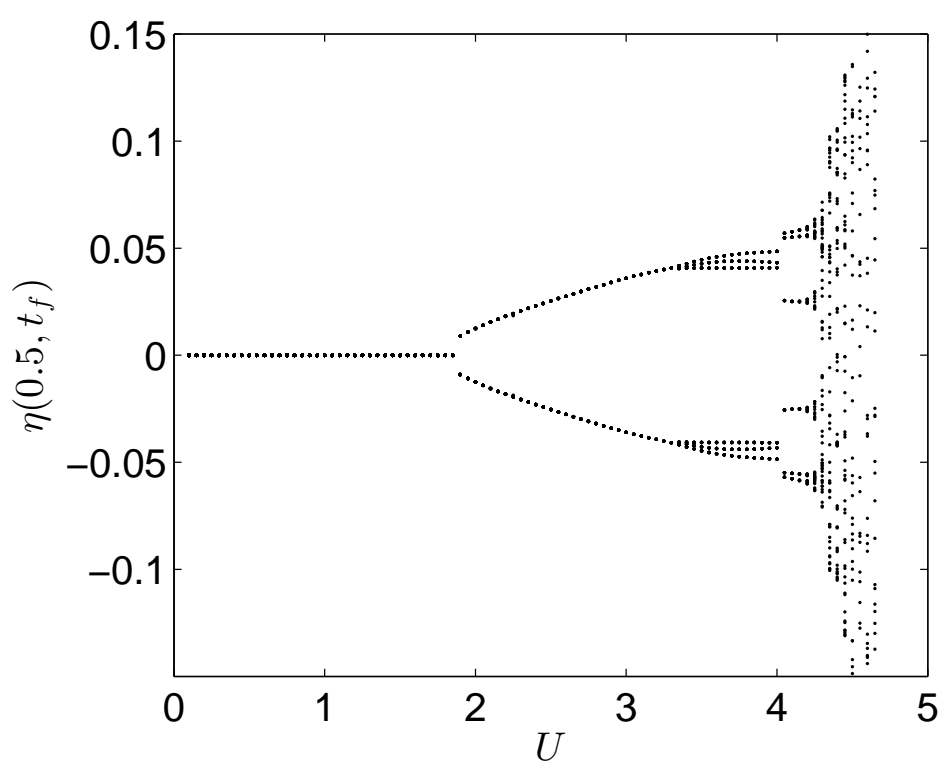

Figure 4.6: Bifurcation diagram of a fixed-fixed beam with cubic spring baffle located at $\xi_{b}=0.45$

region the bifurcation diagram was kept empty. For the already mentioned range of velocity, when we compare both cases of symmetric and asymmetric loading, a symmetric loading shows steady response throughout the velocity range, whereas at some points the asymmetric loading shows an unstable and more chaotic response.

\subsection{Modelling of baffle as a tri-linear spring}

As we discussed in the section 4.4, a cubic spring model closely resemble the free-gap mathematically. But to recognize a physical model of free-gap exactly, the following tri-linear spring model has been used:

$$
\tilde{f}\left(\eta_{b}\right)=\kappa\left[\eta_{b}-\frac{1}{2}\left(\left|\eta_{b}+d_{g}\right|-\left|\eta_{b}-d_{g}\right|\right)\right]
$$

When compared with a cubic spring, a tri-linear spring with proper selection of free-gap $d_{g}$ and spring stiffness $\kappa$ defines better approximation to a physical baffle. A schematic diagram of tri-linear function is shown in the Fig. 4.9. It is clear from the figure that the restrained force corresponds to the deflection ranges from $-d_{g}$ to $+d_{g}$ is zero. But beyond these limits, it shows a linear variation of restrained force with respect to the deflection. The magnitude of restrained force depends on the value of $\kappa$. When we increase the $\kappa$ to higher value, the restrained force also become very high, and the spring will represent the physical rigid baffle. Note that the value of $d_{g}$ mentioned in the figure is taken only for demonstration purpose. The forcing vector $\boldsymbol{F}$ of Eq. 3.20 is defined as $\boldsymbol{F}=\left[\boldsymbol{F}_{1}^{\mathrm{T}}, \boldsymbol{F}_{2}^{\mathrm{T}}\right]^{\mathrm{T}}$. Where $\boldsymbol{F}_{1}$ is a zero vector of order $N \times 1$ and for a tri-linear spring $\boldsymbol{F}_{2}$ is given below;

$$
\boldsymbol{F}_{2}=\kappa\left[\eta_{b}-\frac{1}{2}\left(\left|\eta_{b}+d_{g}\right|-\left|\eta_{b}-d_{g}\right|\right)\right] \boldsymbol{\phi}, \quad \text { where } \eta_{b}=\boldsymbol{\phi}^{\mathrm{T}} \boldsymbol{q}
$$




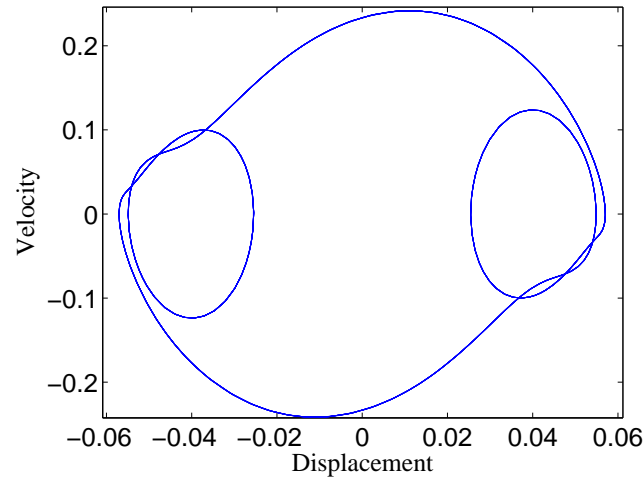

(a)

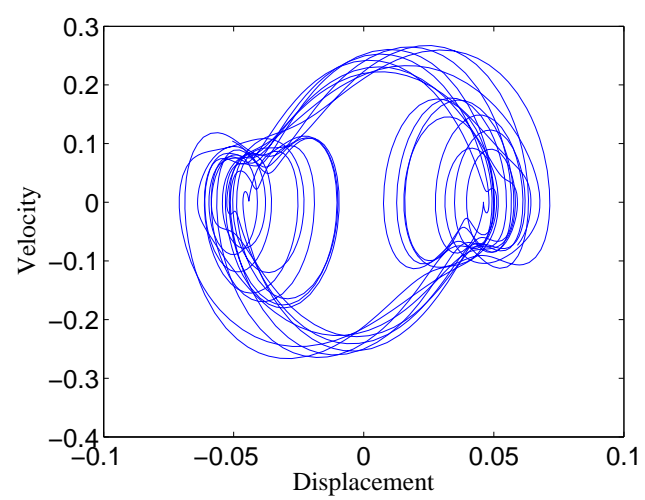

(c)

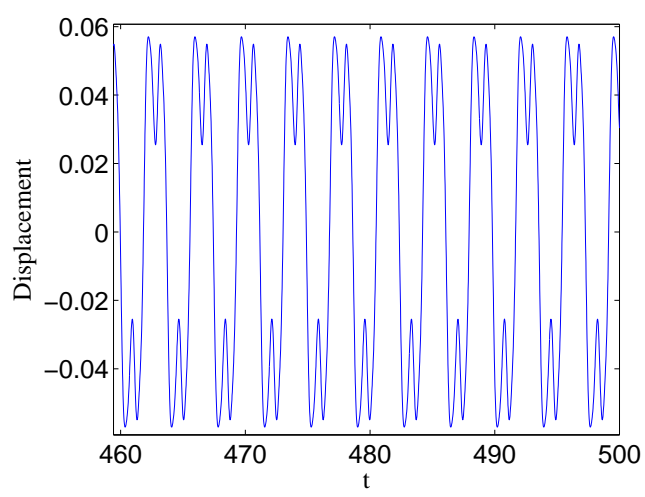

(b)

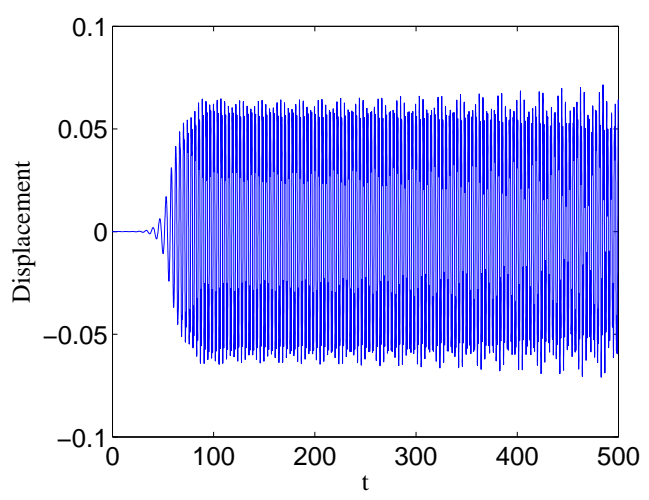

(d)

Figure 4.7: Phase plots and time responses associated with the bifurcation diagram of Fig. (4.6): (a) and (b) are for $U=4.05$, and (c) and (d) are for $U=4.3$ 


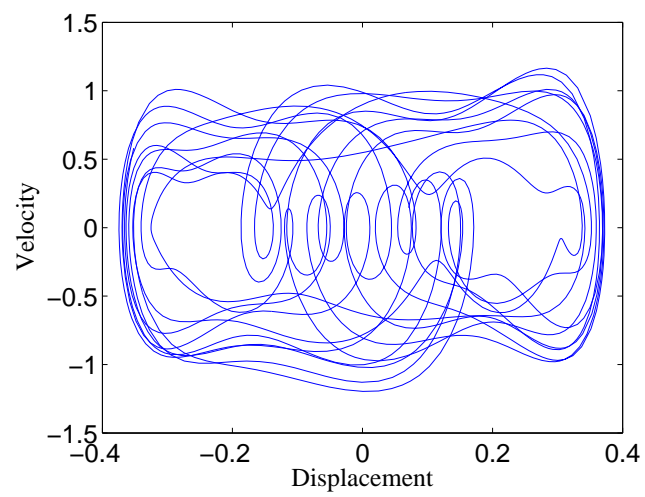

(a)

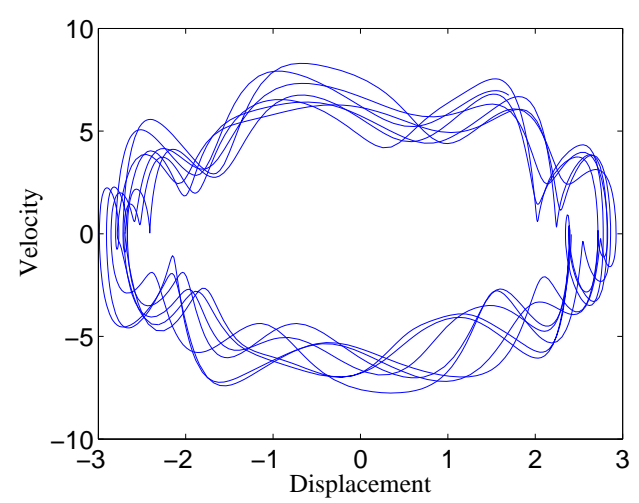

(c)

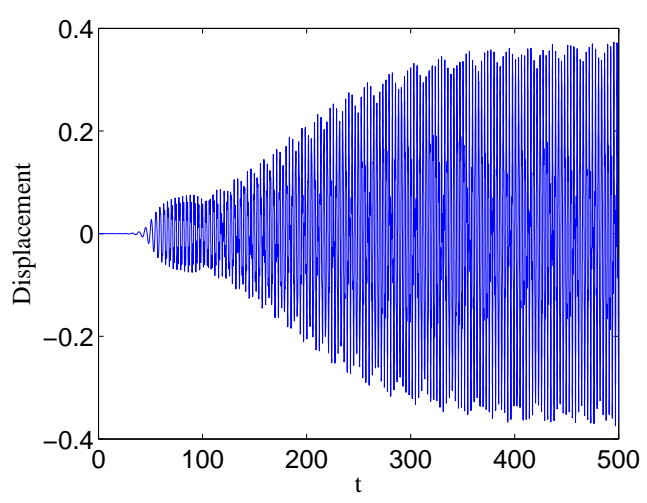

(b)

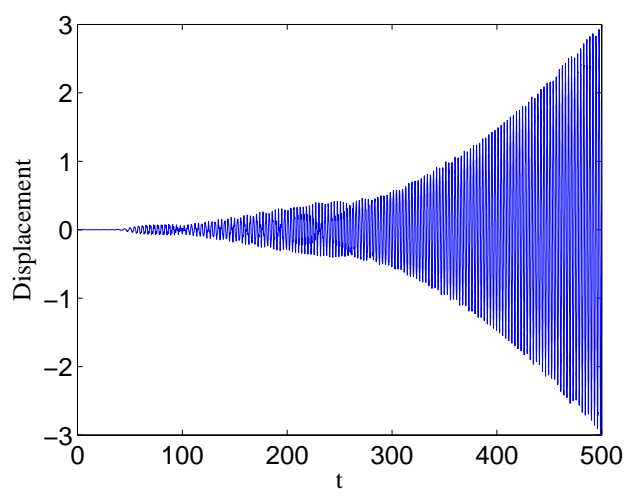

(d)

Figure 4.8: Phase plost and time responses associated with the bifurcation diagram of Fig. 4.6: (e) and (f) are for $U=4.65$, and (g) and (h) are for $U=4.75$.

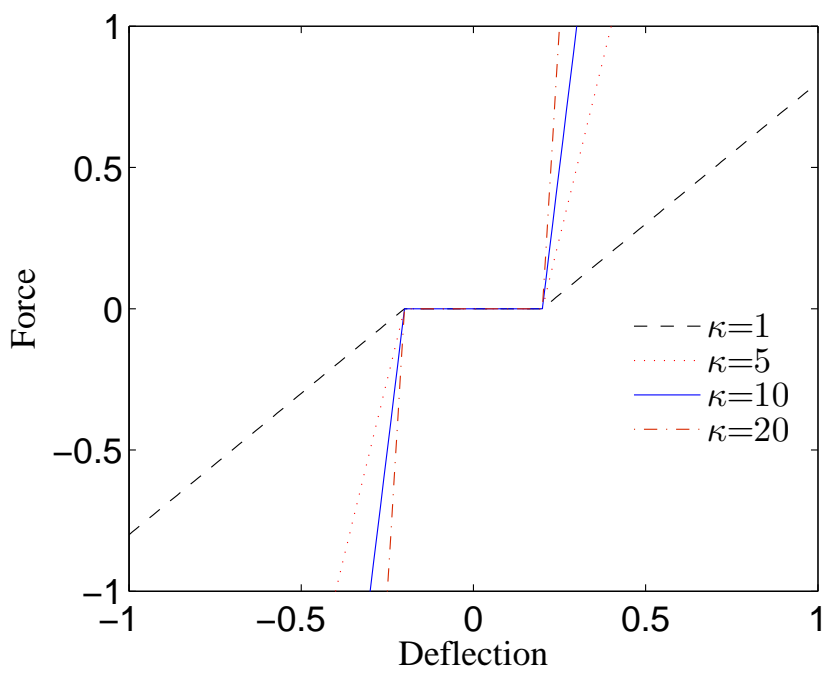

Figure 4.9: Demonstration of tri-linear spring force along with the deflection at different $\kappa$ values. 


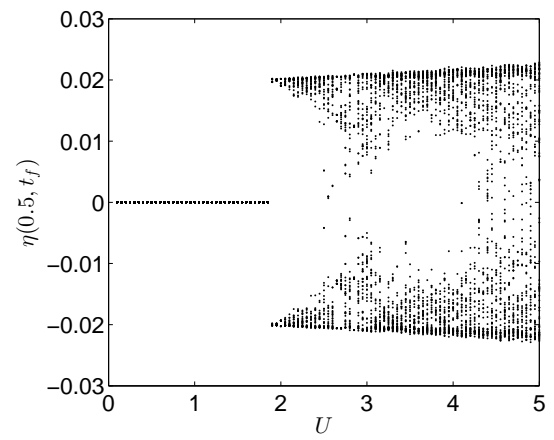

(a)

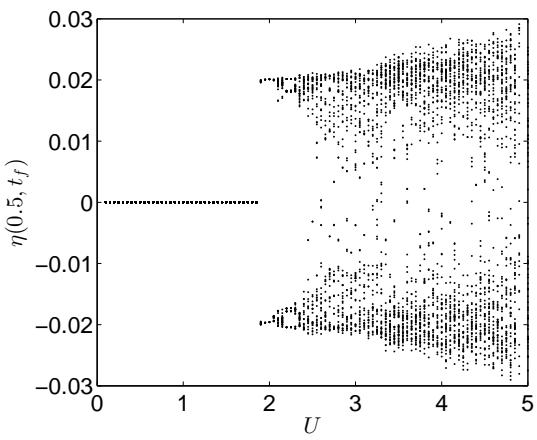

(b)

Figure 4.10: The bifurcation diagram of a fixed-fixed beam with tri-linear spring as baffle. It shows the mid-span $(\xi=0.5)$ amplitude of oscillation for a time range of $t_{f}(t \in[400,500])$. (a) spring kept at the mid-span of the tube $\left(\xi_{b}=0.5\right)$, (b) spring kept at $\xi_{b}=0.45$.

\subsubsection{Bifurcation due to symmetric loading}

Figure. 4.10 shows the bifurcation diagram of fixed-fixed beam with a tri-linear spring kept at the mid span $\left(\xi_{b}=0.5\right)$ of the beam. The displacement of the system at $\xi=0.5$ is measured with respect to the non-dimensional velocity $U$ with default parameters. The stiffness of the spring is assumed as $\kappa=1000$, and the free-gap between baffle and the tube is taken as $d_{g}=0.02$. For a flow with velocity ranging from 0 to 2 , the system oscillate with very less amplitude. But a further increase in the velocity makes the systems to oscillate with an amplified amplitude, later onward the the amplitude become finite and steady. It is also observed that the system is highly chaotic after the velocity $U=2$.

Figure. 4.11 shows the phase plot and time response at different velocities. At $U=2$ (see Fig. 4.11(a)-(b)) even though the systems amplitude is less it shows an increasing trend, so this operating velocity range is not recommended for the system. When the velocity has been increased to $U=2.1$, the system becames chaotic with a finite displacement. But it can be observed that for a velocity $U=2.6$ and $U=2.85$, the system is not chaotic, Fig. 4.11(e)-(f) and Fig. 4.11(g)-(h) shows the corresponding time response. But in between $U=2.6$ and $U=2.85$ highly chaotic responses are observed. Likewise we can expect other high velocities at which the system oscillate with a steady and non-chaotic way. In an ideal case one can find these points to operate the heat exchanger system where the system oscillate with an amplified steady state.

\subsubsection{Bifurcation due to asymmetric loading}

Like in section 4.4.2, for an asymmetric loading the tri-linear spring has been placed at $\xi_{b}=0.45$ and the amplitude of the oscillation at $\xi=0.5$ is measured. The diagram (Fig. 4.10(b)) shows that after $U=2$ the system is completely chaotic. Figure 4.12 shows the phase plot and the time response at different flow velocities as mentioned in the caption. When we compare an asymmetric loading with a symmetric loading, there no difference in the critical velocity. But because of the offsetting of the spring from the mid-span of the tube, the amplitude of the oscillation at the mid-span is more than that of a symmetric loading. 


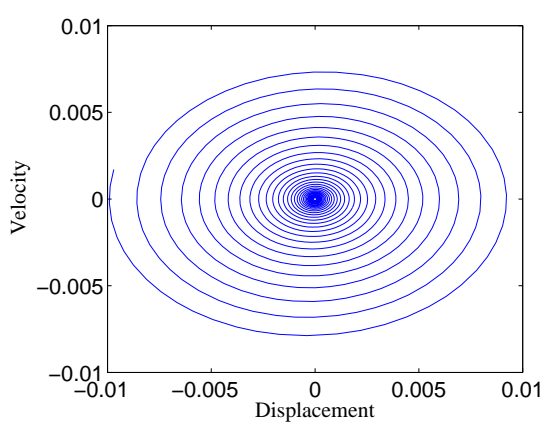

(a)

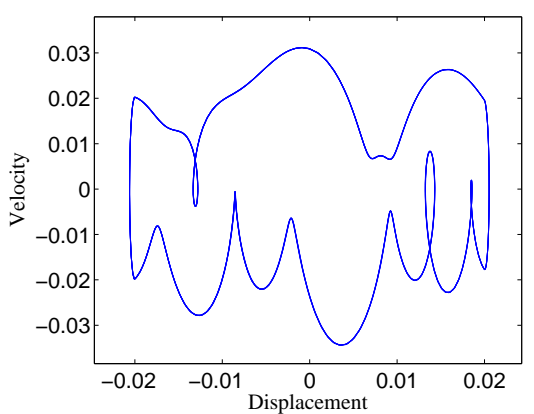

(c)

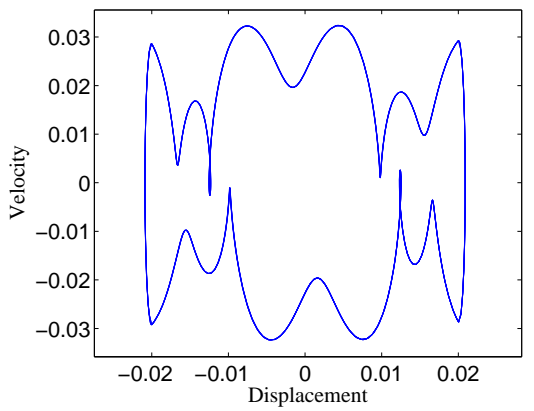

(e)

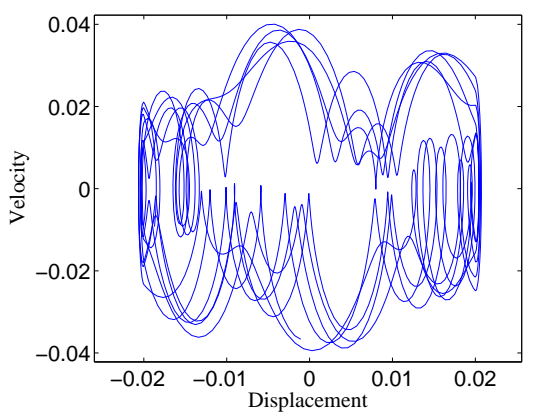

(g)

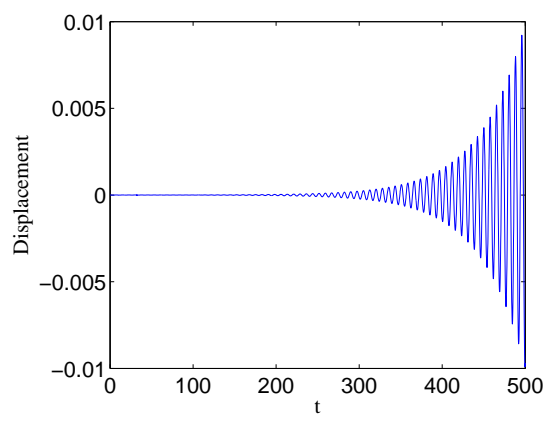

(b)

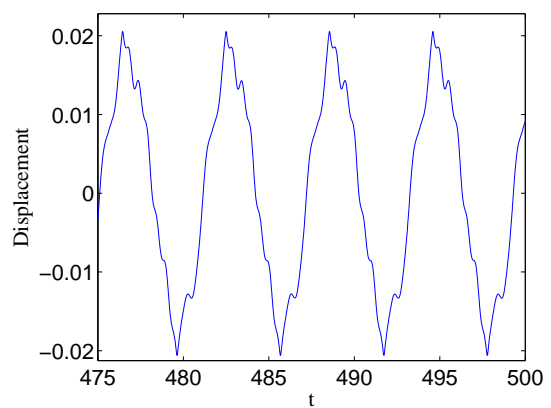

(d)

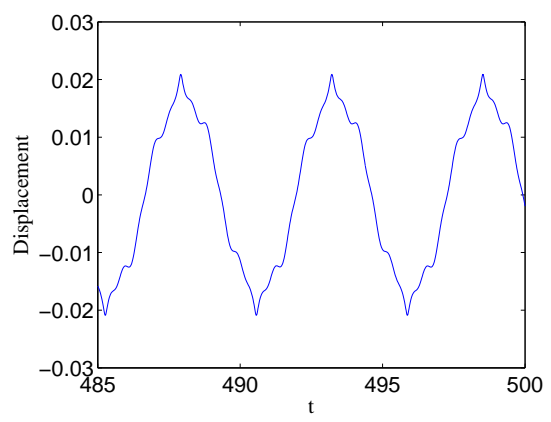

(f)

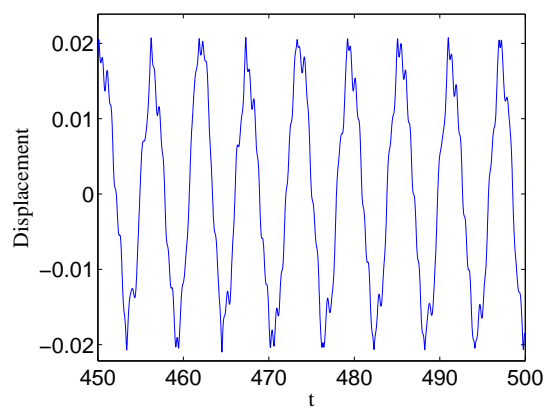

(h)

Figure 4.11: Phase plots and time responses associated with the bifurcation diagram of Fig. 4.10(a), (a) and (b) are for $U=2,(\mathrm{c})$ and (d) are for $U=2.6$, (e) and (f) are for $U=2.85$, and (e) and (f) $U=2.9$. 


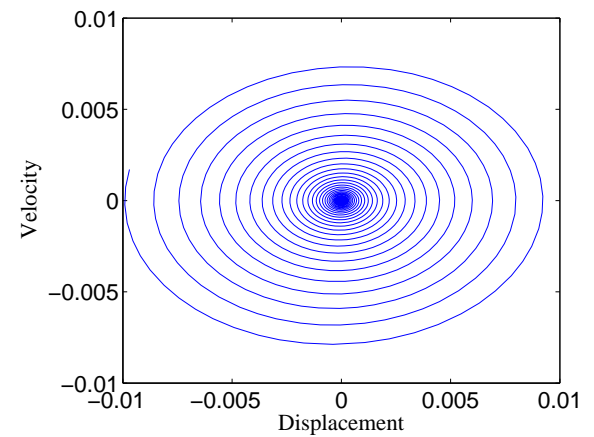

(a)

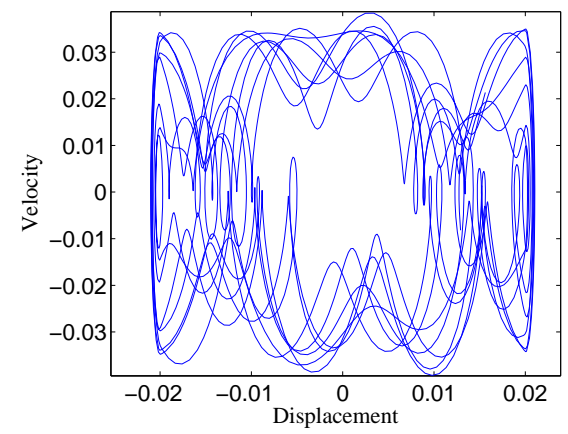

(c)

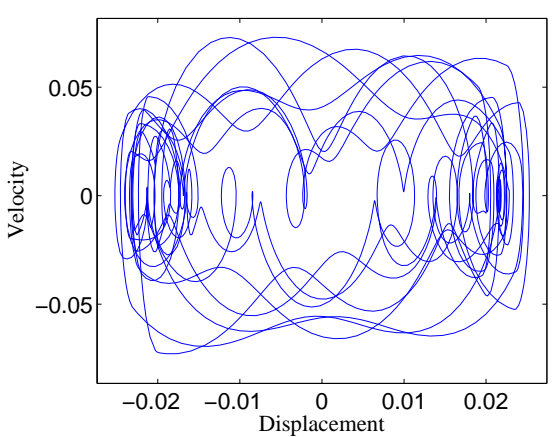

(e)

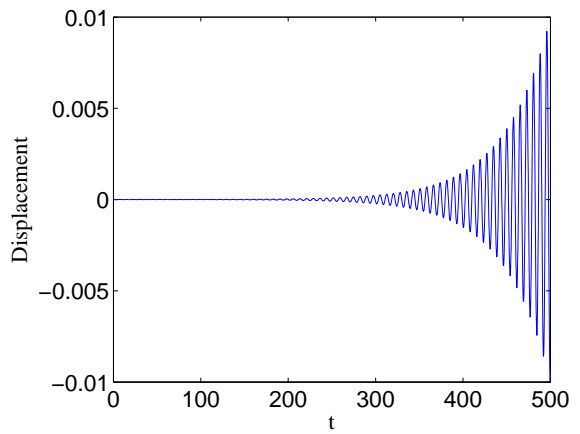

(b)

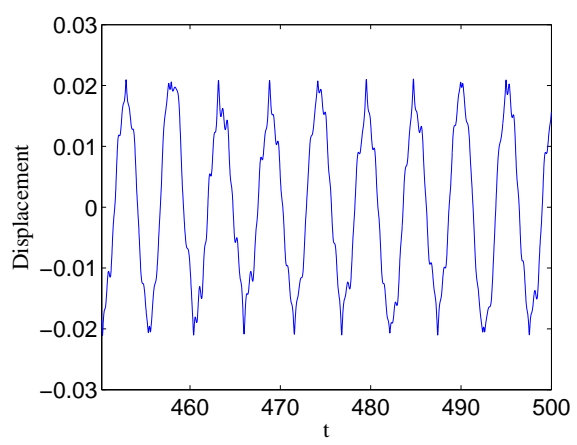

(d)

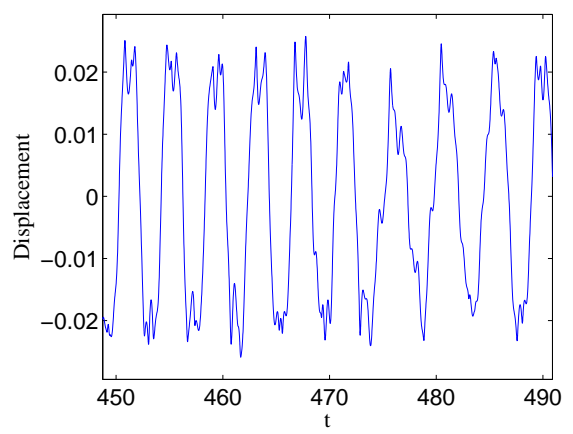

(f)

Figure 4.12: Phase plots and time responses associated with the bifurcation diagram of Fig. 4.10(b): (a) and (b) are for $U=2,(\mathrm{c})$ and (d) are for $U=3$, and (e)and (f) are for $U=4$. 


\section{Chapter 5}

\section{Fixed-free beam modelling}

As discussed in the section 1.2, the chaotic and quasi-periodic vibration of the beam with fixed-fixed boundary conditions may lead to the generation of cantilever boundary conditions once the support fails. But the free end is allowed only to oscillate within a small free-gap. So the loose supports at the free end is replaced with a high stiffness spring. Also the influence of already existing loose support at the centre of the beam has to be studied. So in this chapter we will model the system as an Euler-Bernoulli cantilever beam with two loose supports, one at the centre and the other one is at the free end.

\subsection{Shape functions}

Like fixed-fixed beam, the the contribution of higher modes on the systems response in negligible, so maximum five modes have been considered to approximate the series solution (Eq. (3.14)). The shape function of a unit length fixed-free beam $\phi(\xi)$ (Eq. (3.14)) is defined as below

$$
\phi_{i}(\xi)=\left(\cosh \lambda_{i} \xi-\cos \lambda_{i} \xi\right)-\sigma_{i}\left(\sinh \lambda_{i} \xi-\sin \lambda_{i} \xi\right)
$$

where;

$$
\sigma_{i}=\frac{\cos \lambda_{i}+\cosh \lambda_{i}}{\sin \lambda_{i}+\sinh \lambda_{i}}
$$

and the dimensionless eigenvalues are given by

$$
\boldsymbol{\lambda}=[1.8751,4.7300,7.8532,10.9956,14.1372] .
$$

\subsection{Hopf bifurcation}

In this section the critical velocities of a fixed-free beam has been studied. Like we discussed in section 4.2 , one can arrive at the same equation as given in the Eq. 4.5 for a cantilever beam

$$
\ddot{q}_{i}(\tau)+\left(\beta_{1}+\beta_{2} U\right) \dot{q}_{i}(\tau)+\beta_{3} q_{i}(\tau)+\beta_{4} U^{2} q_{i}(\tau-T)=0
$$




\begin{tabular}{lll}
\hline Modes & $U_{H}$ & $\omega_{i}$ \\
\hline 1 & 1.785 & 0.824 \\
2 & 11.186 & 5.166 \\
3 & 31.320 & 14.465 \\
4 & 61.374 & 28.346 \\
5 & 101.456 & 46.858 \\
\hline
\end{tabular}

Table 5.1: Critical dimensionless flow velocity with non-dimensional natural frequency for a fixed-free beam corresponds to the mode shape.

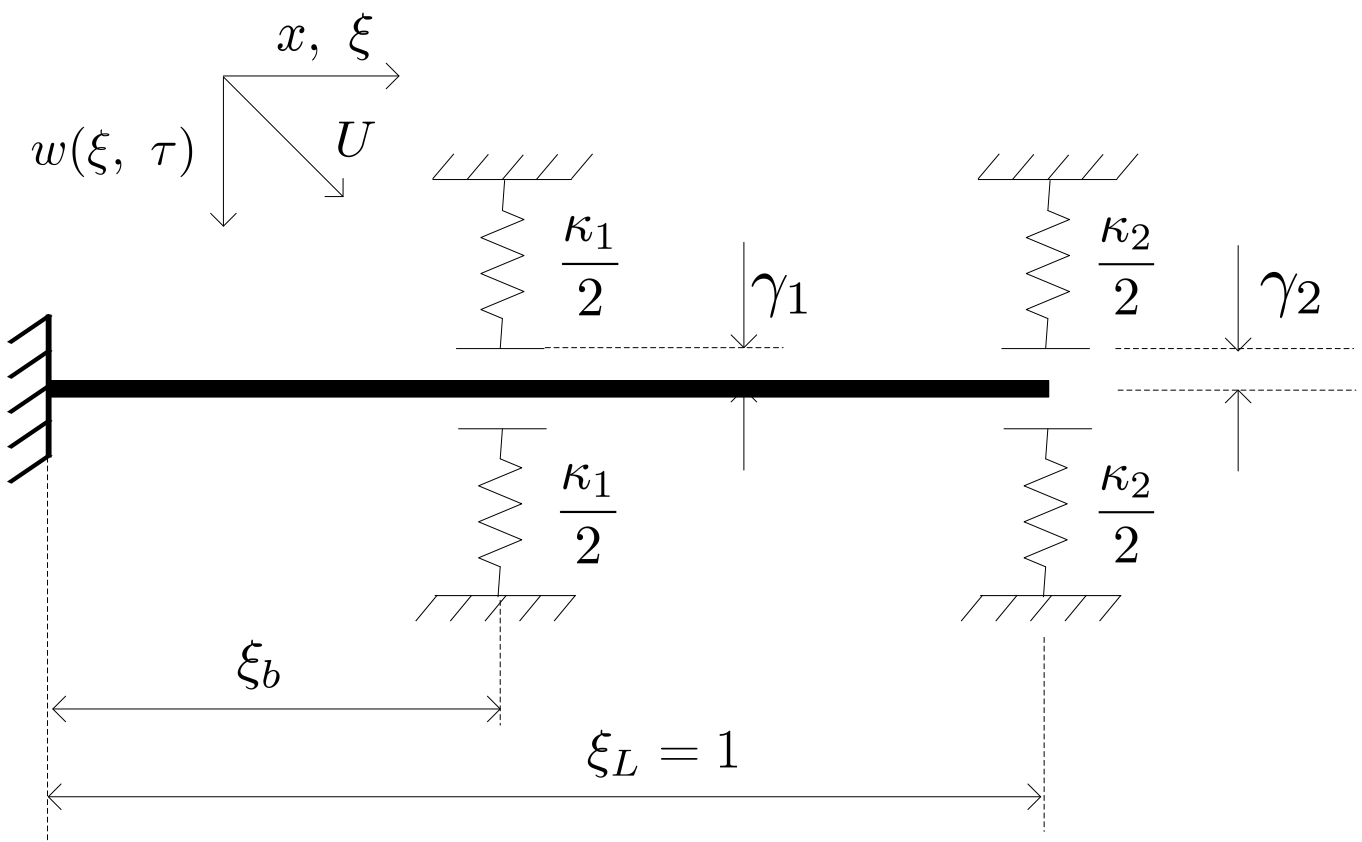

Figure 5.1: The schematic representation of a fixed-free beam with mid-span and tip loose supports.

where $\beta_{1}=0.0145 v_{i}, \beta_{2}=0.00524, \beta_{3}=0.76 v_{i}^{2}$, and $\beta_{4}=0.026$. But here the values of $v_{i}$ depends on non-dimensional eigenvalues $\lambda_{i}$, that is $v_{i}=\left(\frac{\lambda_{i}}{\lambda_{1}}\right)^{2}$. So one can get the following table 5.1 for cantilever beam. As we discussed in section 4.2 , here the fist critical velocity of cantilever beam is $U_{H}=1.785$. Since the remaining critical velocities shown in the table are due to the decoupled equation, also it is clear that after the first Hopf bifurcations the decoupled system become coupled, thereby the rest of the critical velocities also will change.

In section 5.3, the forcing term in the Eq. (3.19) is modified to account the additional loose support. In section 5.4, we model both the loose supports as a cubic spring. In section 5.5 both loose supports are replaced with two tri-linear springs.

\subsection{Equation of motion}

Equation (5.5) represent the model of the cantilever beam with two forcing terms represented by $\tilde{f}_{\text {tip }}\left(\eta_{t i p}\right)$ and $\tilde{f}_{b}\left(\eta_{b}\right)$. Where $\xi_{\text {tip }}=1$ is the location of the tip loose support and $\xi_{b}=0.5$ corresponds 
to the support at the middle.

$$
\begin{gathered}
\frac{1}{1-\beta} \ddot{q}_{i}(\tau)+\left(\zeta+\frac{\tilde{U} C_{D}}{4 \pi \tilde{m}}\right) \dot{q}_{i}(\tau)+\left(\frac{\lambda_{i}}{\lambda_{1}}\right)^{2} q_{i}(\tau) \\
-\frac{\tilde{U}^{2}}{8 \pi^{2} \tilde{m}} \frac{\partial C_{L}}{\partial \eta} q_{i}(\tau-T)+\phi_{i}\left(\xi_{b}\right) \tilde{f}\left(\eta_{b}\right)+\phi_{i}\left(\xi_{t i p}\right) \tilde{f}_{t i p}\left(\eta_{t i p}\right)=0 \quad i=1,2, \ldots, N
\end{gathered}
$$

Where the shape function $\phi_{i}$ is the mode shape of the cantilever beam (see section 5.1). Also the force vector $\boldsymbol{F}$ in Eq. (3.20) changes to $\boldsymbol{F}=\boldsymbol{F}_{b}+\boldsymbol{F}_{t i p}$.

\subsection{Loose supports as a cubic spring}

In this section, we model the loose supports as cubic springs (see Fig. 5.1). Three different cases have been considered by taking different combinations of spring stiffness. In the first case, we consider $\kappa_{1}=1000$ and $\kappa_{2}=2000$, in the second case, we consider $\kappa_{1}=1500$ and $\kappa_{2}=1500$ and for the third case, we take $\kappa_{1}=2000$ and $\kappa_{2}=1000$. The bifurcation diagram with reference to the tip $(\xi=1)$, and with reference to the mid-span $(\xi=0.5)$ have been drawn. Figure 5.2 shows the bifurcation diagram of the three cases as mentioned above. For all the above mentioned cases, the Hopf bifurcation occurs at $U \approx 1.75$ as shown in the Fig. 5.2. It can be seen from table. 5.1 that the critical velocity velocity $U=1.785$ approximately matches with the that obtained after the numerical integration $(U \approx 1.75)$. This shows that the effect of the cubic springs on the systems response for $U<1.75$ is negligible.

The tip deflection (Fig. 5.2 (b), (d), and (f)) shows that, the first post-Hopf bifurcation occurs at a velocity $U=3.75$, where as the first post-Hopf bifurcation of the mid-span occurs only at $U=4.4$ (see Fig. 5.2(a), (c) and (e)). It is interesting to note that, once the velocity reaches $U=4.85$, both points (tip and mid) of the system become chaotic. Even though the system is chaotic after $U=4.85$, it oscillate with a finite amplitude.

It can be observed that, the deflection of the beam is less when we keep the high stiffness spring at the tip of the beam as in Fig. 5.2(a)-(b). Fig. 5.2(e)-(f) shows that, even though there is a high stiffen spring at the mid-span, the amplitude of oscillation of mid-span is more than that of all other cases. When we compare Fig. 5.2(b) with Fig. 5.2(f), even though there is a high stiffness spring at the mid-span, the deflection of the mid-span for the case (f) is more than that of case (a). So, a lesser amplitude oscillation is achieved by placing the high stiffness spring at the tip.

The phase plots and the time responses of the beam with respect to the tip of the beam is shown in Fig. 5.3 with reference to Fig. 5.2(a). For a flow velocity $U=3$, the amplitude of the system starts to increase, with respect to time, from zero to a finite value and become steady, we get a limit cycle and a sinusoidal response. After the first post-Hopf bifurcation, for example at $U=4$, the tube oscillate with high amplitude and hits on the spring and oscillate six times locally (see Fig. 5.3(c)-(d)). Figure 5.3(e)-(f) shows that the system shows a chaotic oscillation at a flow velocity $U=5$.

In Fig. 5.4, we shows the phase plots and the time responses corresponds to the mid-span of the tube (with reference to Fig. 5.2(b)). It can be observed that at $U \approx 4.4$ there is stage where both system displacement and system velocity goes near to zero (see Fig. 5.4 (a)-(b)). When we increase 
the flow velocity further, the system oscillate locally around the stable point (system displacement and system velocity is zero) with a lesser amplitude (see Fig. 5.4 (c)-(d)). It can also be observed that, for steady state oscillation, the mid point of the beam oscillate with a maximum six peak points, after $U=4.48$ the system become chaotic. If we divide the velocity ranges into three regions: the stable state, the post-Hopf bifurcation and the chaotic region, it has been observed that the entire system response is same. That means the the system is stable before $U=1.75$, a post-Hopf bifurcation stage in between $U=1.75$ and $U=4.85$, and a chaotic region after $U=4.85$.

\subsection{Loose supports as a tri-linear spring}

As we discussed before, a tri-linear spring can replicate the free-gap between the baffle and the tube of a heat exchanger. Moreover if the stiffness of the spring is high, it can model as a physical rigid baffle. In this section we study the effect of the free-gap in the system response.

With reference to Fig, 5.1, we consider three different combinations of $\gamma_{1}$ and $\gamma_{2}$ after keeping the stiffness of both springs $\kappa_{1}$ and $\kappa_{2}$ to a constant value (1000). Let us assume that the free-gap at the mid-span $\gamma_{1}=0.02$ is a constant and take three different cases for the free-gap at the tip of the beam. The first one; free-gap at the tip is less than the free-gap at mid-span. The second one; equal free-gap at both tip and mid-span. The last one; free-gap at the tip is greater than the free-gap at mid-span.

Figure 5.5 shows the bifurcation diagram of a cantilever beam at both locations $\xi=0.5$ (see Fig. 5.5(a), (c), and (e)) and $\xi=1$ (Fig. 5.5(b), (d), and (f)). It is clear from the Fig. 5.5 that the the critical velocity is $U \approx 1.75$. When we increase the flow velocity beyond $U=1.75$, the system become chaotic for all the cases. This is same for all the cases mentioned in the figure. One can observe that the maximum amplitude of oscillation is controlled by the free-gap located at the tip of the beam, so the maximum allowed amplitude is less than the tip free-gap.

When we observe Figs. 5.5(a), (c), and (e), in between flow velocity $U=1.75$ and $U=3.8$, it shows a lower amplitude when compared to response after $U=3.8$. Also at this range, it can be observed that the mid-span of the tube shows an average of four extreme amplitudes. On further increase of the velocity after $U=3.8$, it shows a sudden increase in the amplitude. After $U=3.8$ it can be seen the the mid-span oscillate many times locally near to the zero displacement.

Figure 5.6 shows the phase plots and time responses of the beam with tri-linear spring baffles for free-gap $\gamma_{1}=0.02$ and $\gamma_{2}=0.03$ (see Figs. 5.5(e)-(f)). Figures 5.5(a)-(d) are corresponds to the mid-span amplitude and Figures 5.5(e)-(h) are corresponds to the tip amplitude. All time responses shows that the system is chaotic for any velocity beyond $U=1.75$. 


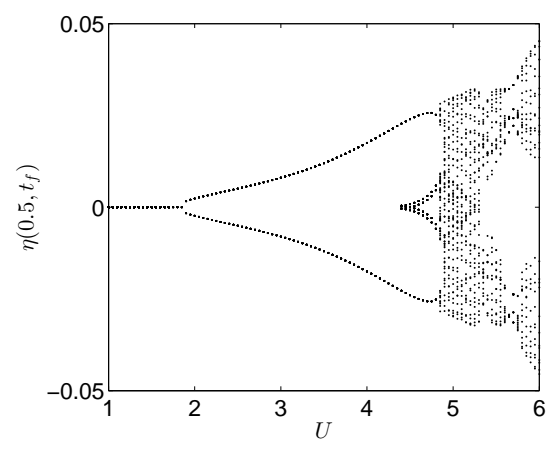

(a)

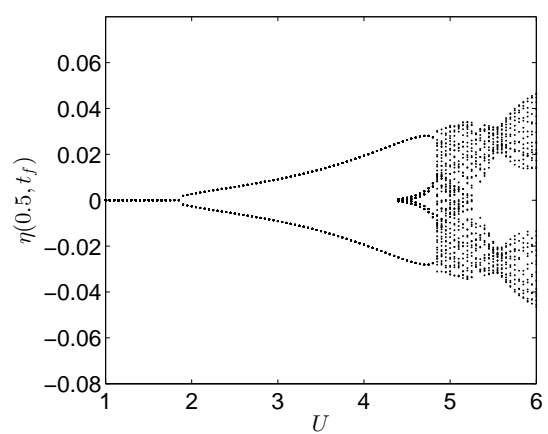

(c)

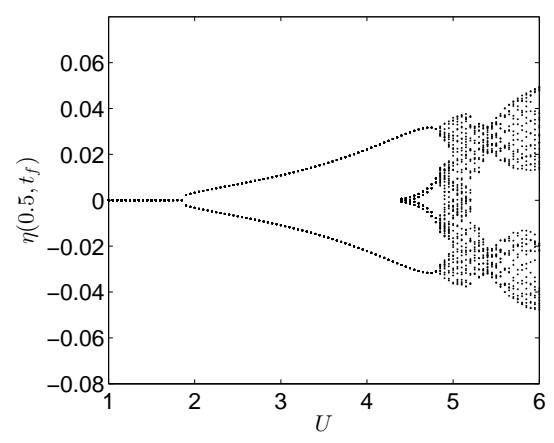

(e)

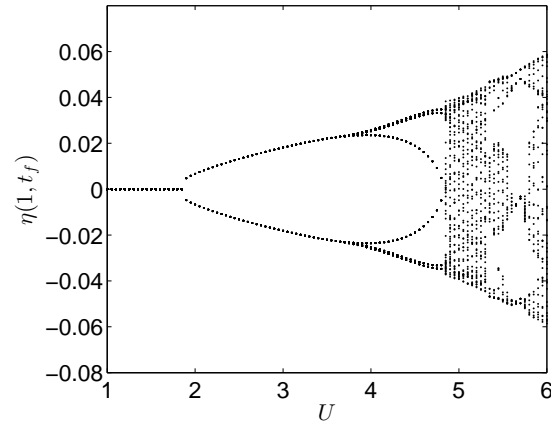

(b)

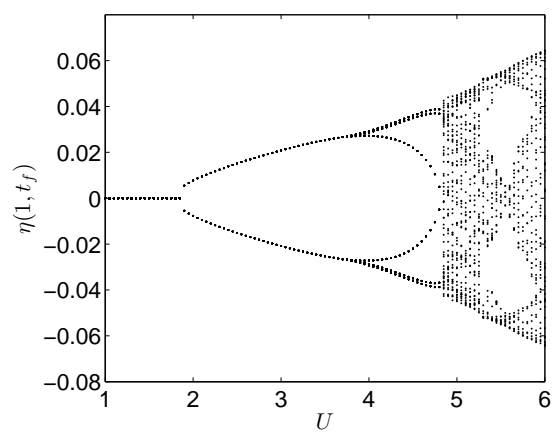

(d)

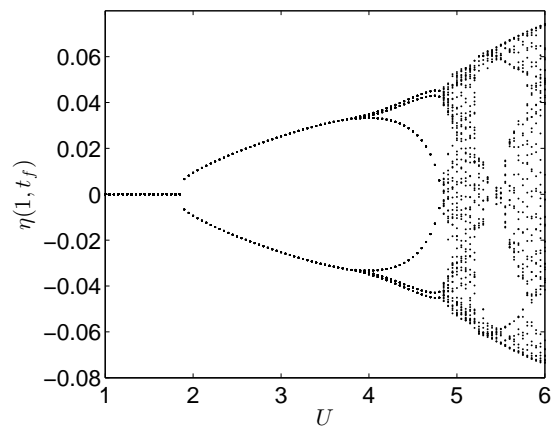

(f)

Figure 5.2: The bifurcation diagram of cantilever beam with two cubic springs. The peak values of the response are plotted, (a), (c), and (e) are at $\xi_{b}=0.5$, and (b), (d), and (f) are at $\xi_{t i p}=1$. The stiffness values, (a) and (b) are for $\kappa_{1}=1000$ and $\kappa_{2}=2000$, (c) and (d) are for $\kappa_{1}=1500$ and $\kappa_{2}=1500$ and (e) and (f) are for $\kappa_{1}=2000$ and $\kappa_{2}=1000 . t_{f}$ represents the time at which peak of the amplitude occurs. 


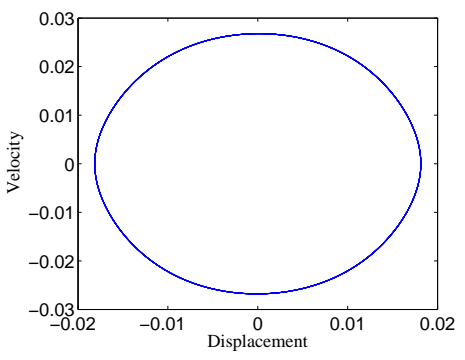

(a)

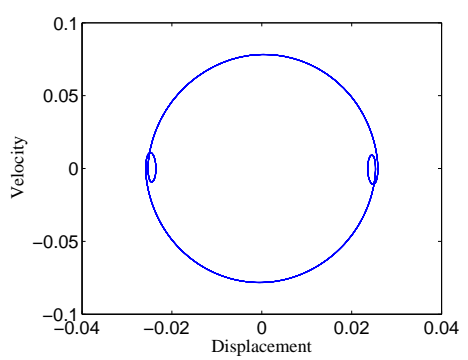

(c)

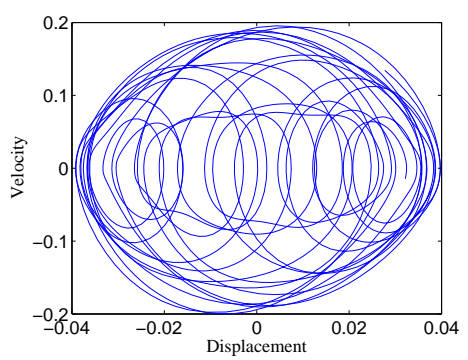

(e)

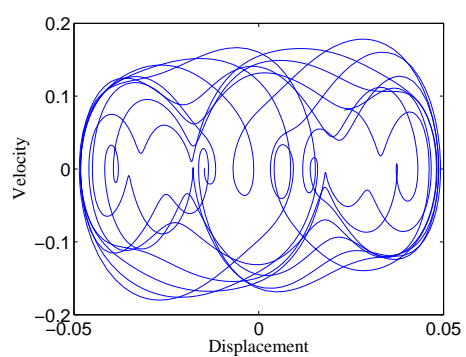

(g)

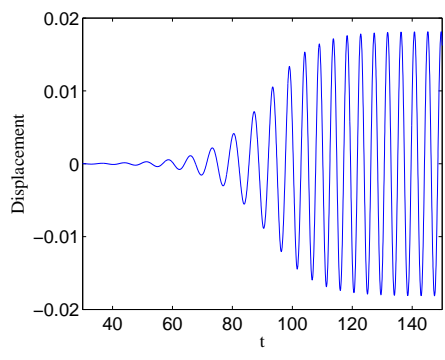

(b)

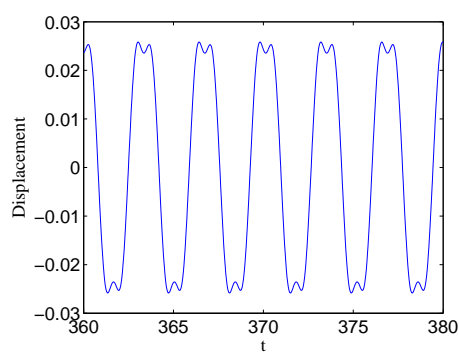

(d)

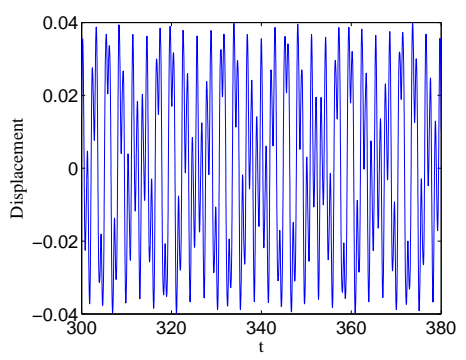

(f)

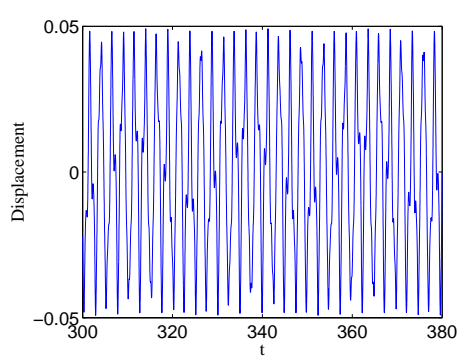

(h)

Figure 5.3: The phase plot and the time response corresponds to the cubic spring stiffness $\kappa_{1}=1000$ and $\kappa_{2}=2000$ at tip of the beam (with reference to Fig. 5.2(a)). The flow velocities are (a) $U=3$, (b) $U=4$, (c) $U=5$, and (d) $U=5.5$ 




(a)

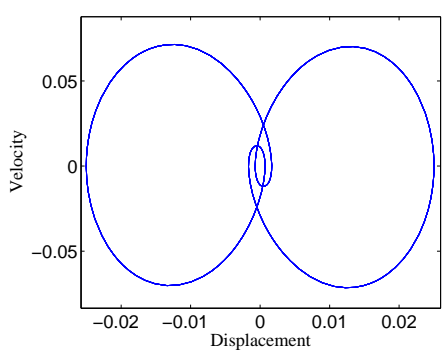

(c)

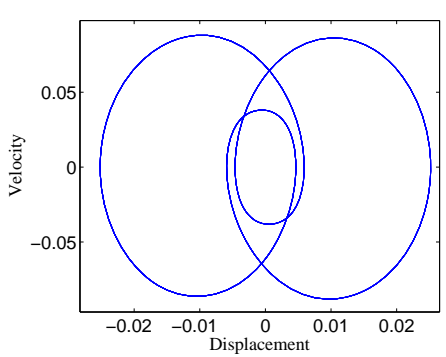

(e)

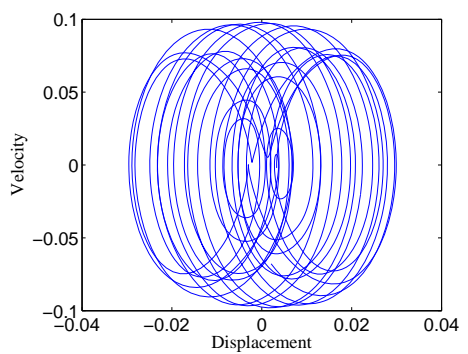

(g)

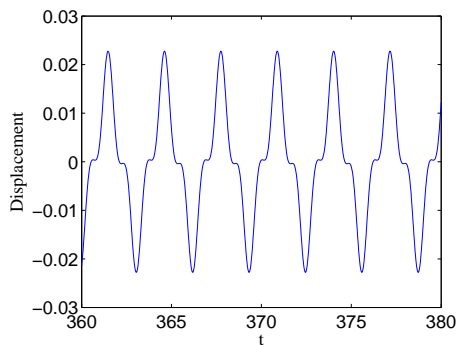

(b)

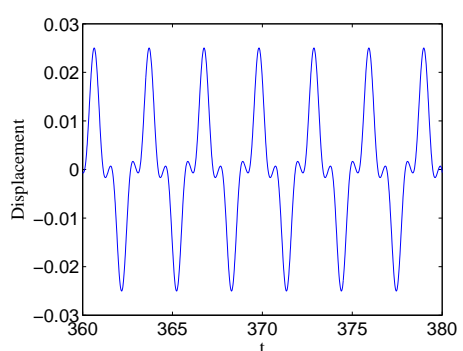

(d)

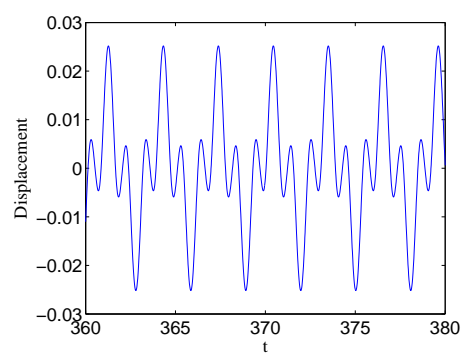

(f)

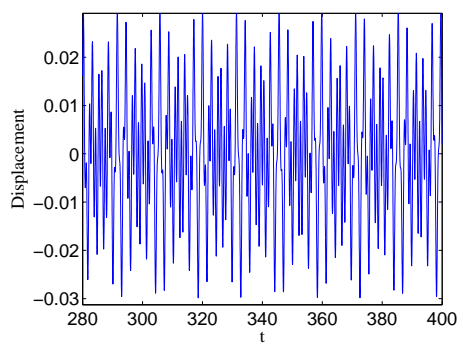

(h)

Figure 5.4: The phase plot and the time response corresponds to the cubic spring stiffness $\kappa_{1}=1000$ and $\kappa_{2}=2000$ at mid span of the tube (with reference to Fig. 5.2(b)). The flow velocities are (a) $U=4.4$, (b) $U=4.6$, (c) $U=4.8$, and (d) $U=5.0$ 


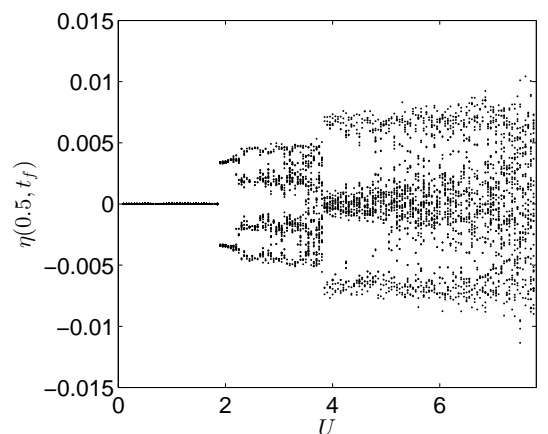

(a)

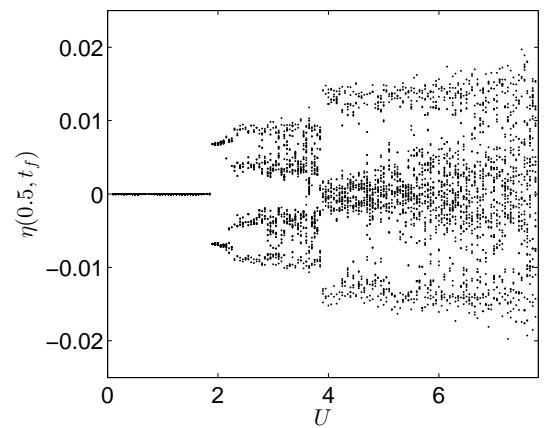

(c)

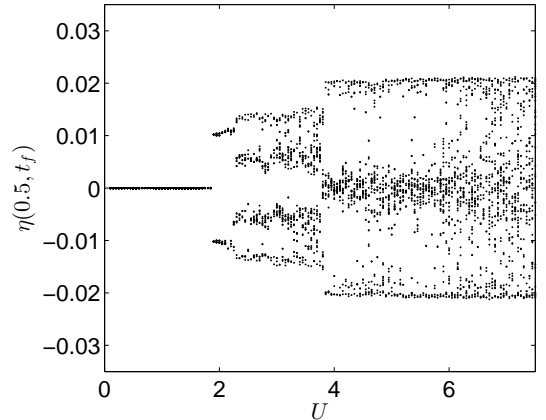

(e)

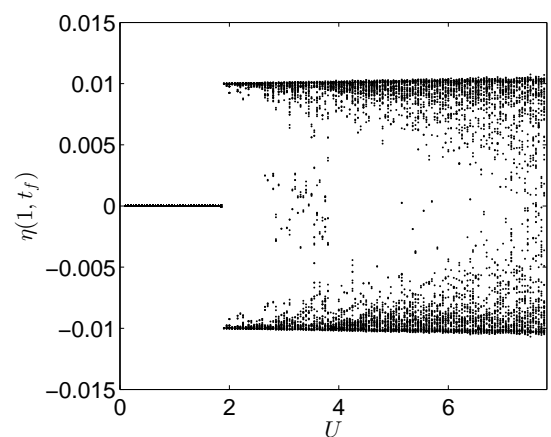

(b)

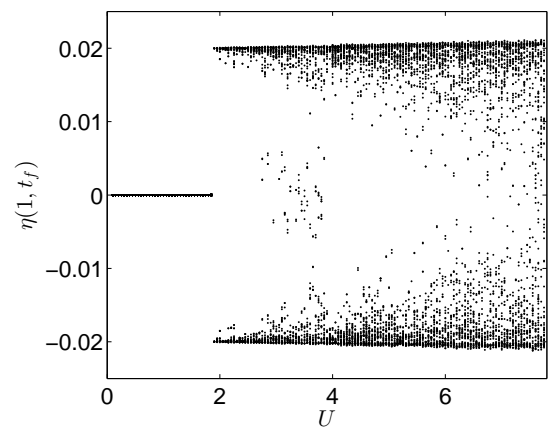

(d)

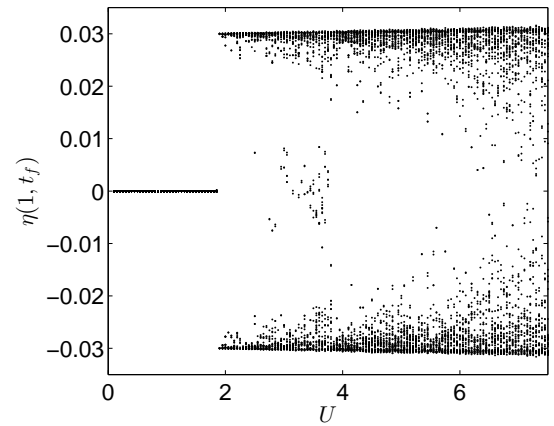

(f)

Figure 5.5: The bifurcation diagram of cantilever beam with two tri-linear springs. (a) and (b) are for $\gamma_{2}=0.01$, (c) and (d) are for $\gamma_{2}=0.02$ and (e) and (f) are for $\gamma_{2}=0.03$. Beam mid-span bifurcation is shown in (a), (c), and (d). Beam tip bifurcation is shown in (b), (d), and (e). $t_{f}$ represents the time at which peak of the amplitude occurs. 


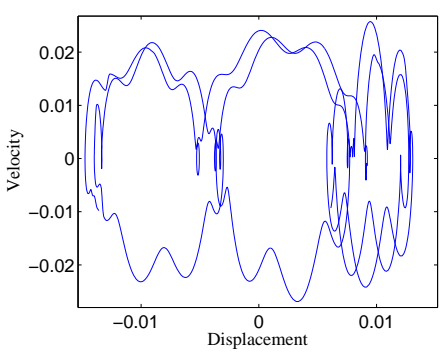

(a)

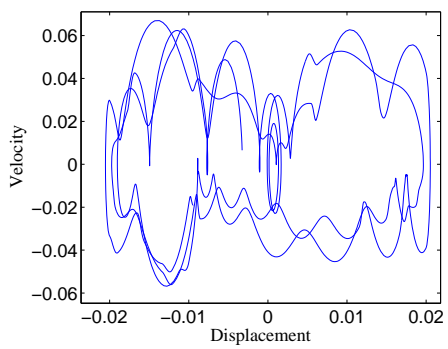

(c)

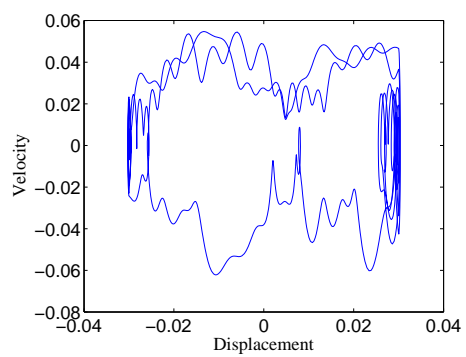

(e)

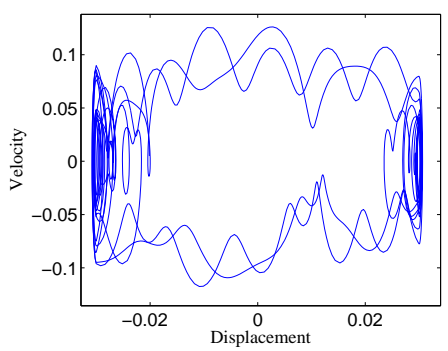

(g)

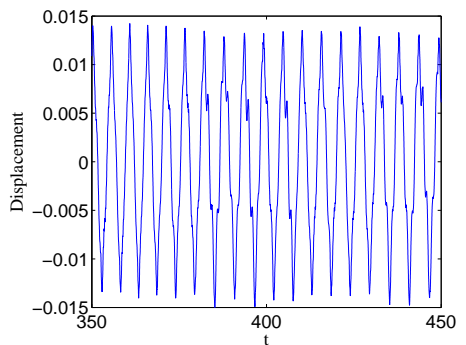

(b)

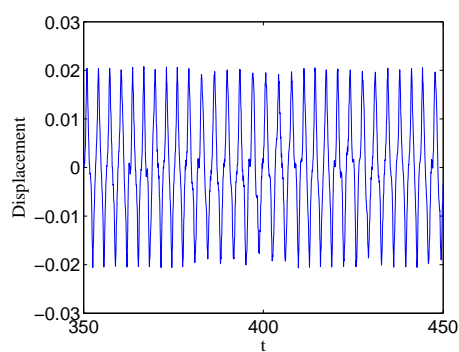

(d)

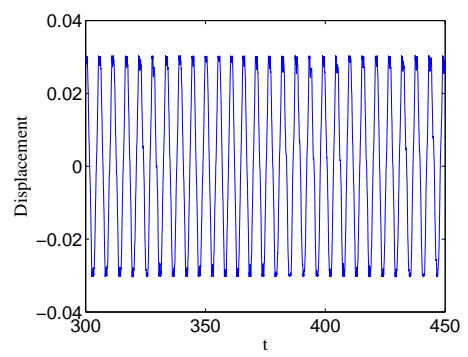

(f)

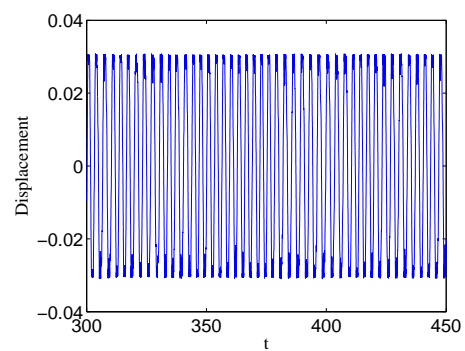

(h)

Figure 5.6: The phase plot and the time response of the tri-linear spring. (a) and (b) for $U=3$ and at $\xi=0.5$, (c) and (d) for $U=5$ and at $\xi=0.5$, (e) and (f) for $U=3$ and at $\xi=1$, (g) and (h) for $U=5$ and at $\xi=1$ 


\section{Chapter 6}

\section{Conclusions}

The intention of this thesis was to study the effect of multiple loose supports on the bifurcation of the heat exchanger tube subjected to cross flow. In this thesis, a fixed-fixed beam model of heat exchanger tube subjected to cross flow has been reviewed. A mathematical model of a cantilever supported with two loose supports has been modelled. Various forces acting on a flexible tube because of the fluid flow has been reviewed. The flow retardation of the fluid was accounted by adding a time delayed term to the displacement. The impact force because of loose supports has been modelled by a cubic spring and a tri-linear spring. The model was approximated to a system second order delay differential equations using Galerkin method by taking five modes of oscillation of a cantilever beam. The obtained system of equation was solved by using Matlab dde solver ("dde23").

The critical fluid flow velocity has been obtained analytically after analysing the Hopf bifurcation of the system. The bifurcation diagram and time response with the flow velocity has been plotted by using the numerical results from the system equations. From the bifurcation diagram we have obtained the critical flow velocity and compared with analytical results. It has been found that, when we increase the flow velocity beyond critical velocity, the system damping has been reduced which amplifies the amplitude of oscillations. A further increase in the velocity makes the system into a negative damping state and it stabilizes the system, this negative damping is because of the generation of nonlinear forces. So this leads to the birth of the limit cycle. A further increase in the velocity leads to the generation several post-Hopf bifurcations. After a particular velocity, the tube impacts on the loose support and the oscillations become more complex and chaotic.

When a loose support has been modelled by a cubic spring; the bifurcation diagram shows that for a velocity less than the critical velocity, the effect of the cubic spring force is negligible. When the velocity has been increased slightly the spring force starts to influences the system response. This shows that the tube has started to contact with the loose support. Now a further increase in the velocity beyond a sufficient velocity, the system response become chaotic.

When a loose support has been modelled by a tri-linear spring, the system become chaotic after the critical velocity. At this stages the bifurcation diagram shows that, a further increase of the maximum amplitude with the velocity is negligible. So the tri-linear spring closely resembles the free-gap of a physical rigid baffle.

It has been found that the spring which was kept at at the free end of the cantilever beam 
has a greater influence on the amplitude of oscillation of the beam. For the same parameters, the deflection of the cantilever model is more when compare with a fixed-fixed beam model. 


\section{References}

[1] M. Pettigrew and D. Gorman. Experimental studies on flow-induced vibration to support steam generator design. Technical Report, Atomic Energy of Canada Ltd., Chalk River, Ontario. Chalk River Nuclear Labs. 1977.

[2] M. Pettigrew and D. Gorman. Vibration of heat exchange components in liquid and two-phase cross-flow. Technical Report, Atomic Energy of Canada Ltd., Chalk River, Ontario. Chalk River Nuclear Labs 1978.

[3] M. Pettigrew and D. Gorman. Vibration of heat exchanger tube bundles in liquid and two-phase cross-flow. ASME, PVP, Flow-induced Vibration Design Guidelines 328, (1981) 97-115.

[4] M. Pettigrew, Y. Sylvestre, and A. Campagna. Vibration analysis of heat exchanger and steam generator designs. Nuclear Engineering and Design 48, (1978) 97-115.

[5] S. Price. A review of theoretical models for fluidelastic instability of cylinder arrays in cross-flow. Journal of Fluids and Structures 9, (1995) 463-518.

[6] R. Ibrahim. Overview of Mechanics of Pipes Conveying Fluids-Part II: Applications and Fluidelastic Problems. ASME Journal of Pressure Vessel Technology 133, (2011) 024,001-1-024,00130.

[7] B. W. Roberts. Low frequency, self-excited vibration in a row of circular cylinders mounted in an airstream. Ph.D. thesis, University of Cambridge 1962.

[8] H. J. Connors and Jr. Fluidelastic vibration of tube arrays excited by cross flow. Proceedings of the ASME, Flow-Induced Vibration in Heat Exchangers, New York, 42-56.

[9] H. J. Connors and Jr. Flow-Induced Vibration and Wear of Steam Generator Tubes. Nucl. Technol., 55(2), (1981) 311-331.

[10] R. Blevins. Fluid elastic whirling of a tube row. ASME Journal of Pressure Vessel Technology 96, (1974) 263-267.

[11] R. Blevins. Fluid damping and the whirling instability of tube arrays. In 3rd National Congress of Pressure Vessel and Piping Technology ASME, San Francisco. American Society of Mechanical Engineers, (1979) 35-39.

[12] M. Wambsganss, C. Yang, and J. Halle. Fluidelastic instability in shell and tube heat exchangers: a framework for a prediction method. Technical Report, Argonne National Lab., IL (USA) 1982 . 
[13] H. Halle, J. Chenoweth, and M. Wambsganss. Flow-Induced Tube Vibration Tests of Typical Industrial Heat Exchanger Configurations. ASME Paper, (1981), 37.

[14] H. Halle, J. Chenoweth, and M. Wambsganss. Flow-induced tube vibration thresholds in heat exchangers from shellside water tests. Technical Report, Argonne National Lab., IL (USA); Heat Transfer Research, Inc., Alhambra, CA (USA) 1984.

[15] S. Chen. Instability mechanisms and stability criteria of a group of circular cylinders subjected to cross-flow. Part I: theory. ASME Journal of Vibration and Acoustics 105, (1983) 51-58.

[16] S. Chen. Instability Mechanisms and Stability Criteria of a Group of Circular Cylinders Subjected to Cross-FlowPart 2: Numerical Results and Discussions. ASME Journal of Vibration and Acoustics 105, (1983) 253-260.

[17] R. Sauve. A computational time domain approach to fluidelastic instability for nonlinear tube dynamics. ASME PVP 328, (1996) 327-335.

[18] F. Eisinger, M. Rao, D. Steininger, and K. Haslinger. Numerical simulation of cross-flow-induced fluidelastic vibration of tube arrays and comparison with experimental results. ASME Journal of Pressure Vessel Technology 117, (1995) 31-39.

[19] R. Violette, M. Pettigrew, and N. Mureithi. Fluidelastic instability of an array of tubes preferentially flexible in the flow direction subjected to two-phase cross flow. ASME Journal of Pressure Vessel Technology 128, (2005) 148-159.

[20] M. Yetisir and D. Weaver. An Unsteady Theory for Fluidelastic Instability in an Array of Flexible Tubes in Cross-Flow. Part I: Theory. Journal of Fluids and Structures 7, (1993) 751766.

[21] M. Yetisir and D. Weaver. An Unsteady Theory for Fluidelastic Instability in an Array of Flexible Tubes in Cross-Flow. Part II: Results and Comparison with Experiments. Journal of Fluids and Structures 7, (1993) 767-782.

[22] C. Taylor, M. Pettigrew, T. Dickinson, I. Currie, and P. Vidalou. Vibration damping in multispan heat exchanger tubes. ASME Journal of Pressure Vessel Technology 120, (1998) 283-289.

[23] G. Rzentkowski and J. Lever. An effect of turbulence on fluidelastic instability in tube bundles: a nonlinear analysis. Journal of Fluids and Structures 12, (1998) 561-590.

[24] D. Weaver, S. Ziada, M. Au-Yang, S. Chen, M. Païdoussis, and M. Pettigrew. Flow-induced vibrations in power and process plant componentsprogress and prospects. Journal of Pressure Vessel Technology 122, (2000) 339-348.

[25] G. Jacquart and N. Gay. Computation of impact-friction interaction between a vibrating tube and its loose supports. Technical Report, Electricite de France (EDF), 92-Clamart (France) 1993.

[26] J. Antunes, F. Axisa, and M. A. Vento. Experiments on VibroImpact Dynamics Under Fluidelastic Instability. Proceedings of the ASME Pressure Vessels Piping, New York 189, (1990) 127-138. 
[27] M. Vento, J. Antunes, and F. Axisa. Tube/support interaction under simulated fluidelastic instability: Two-dimensional experiments and computations of the nonlinear responses of a straight tube. In Winter Annual Meeting of the American Society of Mechanical Engineering, Anaheim, CA, USA, 11/08-13/92. 1992 151-166.

[28] R. Rogers and R. Pick. On the dynamic spatial response of a heat exchanger tube with intermittent baffle contacts. Nuclear Engineering and Design 36, (1976) 81-90.

[29] R. Rogers. Factors associated with support plate forces due to heat-exchanger tube vibratory contact. Nuclear Engineering and Design 44, (1977) 247-253.

[30] Y. Cai and S. Chen. Chaotic vibrations of nonlinearly supported tubes in crossflow. ASME Journal of Pressure Vessel Technology 115, (1993) 128-134.

[31] M. Païdoussis, S. Price, and N. Mureithi. Post-fluidelastic-instability response of a loosely supported tube in an array subjected to water cross-flow. In Winter Annual Meeting of the American Society of Mechanical Engineering, Anaheim, CA, USA, 11/08-13/92. 1992 243-263.

[32] S. M. Fluit and M. J. Pettigrew. Simplified method for predicting vibration and fretting-wear in nuclear steam generators. ASME PVP 420, (2001) 7-16.

[33] M. Païdoussis and G. Li. Cross-flow-induced chaotic vibrations of heat-exchanger tubes impacting on loose supports. Journal of Sound and Vibration 152, (1992) 305-326.

[34] J. De Bedout, M. Franchek, and A. Bajaj. Robust control of chaotic vibrations for impacting heat exchanger tubes in crossflow. Journal of Sound and Vibration 227, (1999) 183-204.

[35] L. Wang and Q. Ni. Hopf bifurcation and chaotic motions of a tubular cantilever subject to cross flow and loose support. Nonlinear Dynamics 59, (2010) 329-338.

[36] S. Price and M. Païdoussis. An improved mathematical model for the stability of cylinder rows subject to cross-flow. Journal of Sound and Vibration 97, (1984) 615-640.

[37] A. Simpson and J. Flower. An improved mathematical model for the aerodynamic forces on tandem cylinders in motion with aeroelastic applications. Journal of Sound and Vibration 51, (1977) 183-217.

[38] S. Price and M. Païdoussis. A single-flexible-cylinder analysis for the fluidelastic instability of an array of flexible cylinders in cross-flow. Journal of Fluids Engineering 108, (1986) 193-199.

[39] S. Granger and M. Païdoussis. An improvement to the quasi-steady model with application to cross-flow-induced vibration of tube arrays. Journal of Fluid Mechanics 320, (1996) 163-184.

[40] A. Ivanov. Analytical methods in the theory of vibro-impact systems. Journal of Applied Mathematics and Mechanics 57, (1993) 221-236. 